\title{
A Supramolecular Approach for Modulated Photoprotection, Lysosomal Delivery and Photodynamic Activity of a Photosensitizer
}

Indranil Roy, ${ }^{\dagger}$ Sharan Bobbala, ${ }^{\ddagger}$ Ryan M. Young, ${ }^{\dagger}, \|$ Yassine Beldjoudi, ${ }^{\dagger}$ Minh T. Nguyen, ${ }^{\dagger}$ M. Mustafa Cetin, ${ }^{\dagger}$ James A. Cooper ${ }^{\dagger}$ Sean Allen, ${ }^{\dagger}$ Ommid Anamimoghadam, ${ }^{\dagger}$ Evan A. Scott, ${ }^{\dagger}$ Michael R. Wasielewski and J. Fraser Stoddart ${ }^{\dagger, \#, \S^{*}}$

${ }^{\dagger}$ Department of Chemistry, ${ }^{\star}$ Department of Biomedical Engineering, "Institute for Sustainability and Energy at Northwestern, Northwestern University, 2145 Sheridan Road, Evanston, Illinois 60208-3113, USA.

"Institute of Molecular Design and Synthesis, Tianjin University, 92 Weijin Road, Nankai District, Tianjin, 300072, China.

\$School of Chemistry, University of New South Wales, Sydney, NSW 2052, Australia.

*E-mail: stoddart@,northwestern.edu

\section{SUPPLEMENTARY INFORMATION}

\section{Table of Contents}

Section A. Materials / General Methods / Instrumentation $\quad$ S2

$\begin{array}{llr}\text { Section B. Synthetic Protocols } & \text { S4 }\end{array}$

$\begin{array}{lll}\text { Section C. NMR Spectroscopy } & \text { S4 }\end{array}$

Section D. Crystallographic Characterization $\quad$ S6

$\begin{array}{llr}\text { Section E. } & \text { Transient Absorption Spectroscopy } & \text { S8 }\end{array}$

$\begin{array}{lll}\text { Section F. Detection of Singlet Oxygen } & \text { S15 }\end{array}$

$\begin{array}{lll}\text { Section G. } & \text { DFT Analysis } & \text { S17 }\end{array}$

$\begin{array}{llr}\text { Section H. } & \text { Cell Experiments } & \text { S24 }\end{array}$

$\begin{array}{lll}\text { Section I. References } & \text { S36 }\end{array}$ 


\section{Section A. Materials / General Methods / Instrumentation}

All chemicals and reagents were purchased from commercial suppliers (Aldrich or Fisher) and used without further purification. $\mathbf{E x B o x} \cdot 4 \mathrm{PF}_{6}$ was synthesized according to previous literature procedures $^{1}$. 5,15-Diphenylporphyrin (DPP) was purchased from TCI chemicals. The synthesis of counterion exchanged product $\mathbf{E x B o x} \cdot 4 \mathrm{Cl}$, and $\mathbf{E x B o x}^{4+} \supset \mathbf{D P P}$ are described in Synthetic Protocols. Thin layer chromatography (TLC) was performed on silica gel 60 F254 (E. Merck). Column chromatography was carried out on silica gel 60F (Merck 9385, 0.040-0.063 mm). Nuclear magnetic resonance (NMR) spectra were recorded on an Agilent Hg400 and Bruker Avance III spectrometers, with working frequencies of 400 and $500 \mathrm{MHz}$, respectively. Chemical shifts were reported in ppm relative to the signals corresponding to the residual nondeuterated solvents $\left(\mathrm{CD}_{3} \mathrm{CN}: \delta 1.94 \mathrm{ppm}\right)$. UV/Vis Absorption spectra were recorded using a UV-3600 Shimadzu spectrophotometer. Steady-state emission spectra were acquired using HORIBA Nanolog spectrofluorimeter equipped with an integrating sphere.

The setup for transient absorption measurements has been described elsewhere. ${ }^{3}$ Photoexcitation pulses at $414 \mathrm{~nm}$ were obtained through a beta barium borate (BBO) crystal doubling the fundamental, and the $575 \mathrm{~nm}$ pulses were generated with a laboratory-constructed optical parametric amplifier. The pulse power for photoexcitation was attenuated to $\sim 1 \mu \mathrm{J} /$ pulse, using neutral density filters. The pump polarization was randomized employing a commercial depolarizer (DPU-25-A, Thorlabs, Inc.) to eliminate any orientational dynamics contributions from the experiment. All the spectra were collected on a commercial spectrometer (Ultrafast Systems, LLC Helios and EOS spectrometers, for fsTA and nsTA, respectively). All samples were stirred to avoid localized heating or degradation effects. The optical density was maintained around 0.5 for all samples.

\section{Data Analysis Time-Resolved Optical Spectroscopy}

Prior to kinetic analysis, the transient absorption data are background/scatter-subtracted and chirpcorrected, and the visible and NIR data sets are spectrally merged (Surface Xplorer 4, Ultrafast Systems, LLC). 
The kinetic analysis was performed using home written programs in MATLAB ${ }^{1}$ and was based on a global fit to selected single-wavelength kinetics. The time-resolution is given as $w=300 \mathrm{fs}$ (full width at half maximum, FWHM); the assumption of a uniform instrument response across the frequency domain and a fixed time-zero $\left(t_{0}\right)$ are implicit in the global analysis.

The kinetic data from multiple different wavelengths are fitted using the global analysis described below. Each wavelength is given an initial amplitude that is representative of the spectral intensity at time $t_{0}$, and varied independently to fit the data. The time/rate constants and $t_{0}$ are shared between the various kinetic data and are varied globally across the kinetic data in order to fit the model(s) described below. We fit the dataset globally to a specified kinetic model and use the resultant populations to deconvolute the dataset and reconstruct species-associated spectra.

For $\mathbf{E x B o x}^{4+} \square \mathbf{D P P}$ at $\mathrm{pH}=7$, we use the species-associated model

$$
\underline{K}=\left(\begin{array}{cc}
-k_{A \rightarrow B} & 0 \\
k_{A \rightarrow B} & 0
\end{array}\right)
$$

with $A(0)=1$ and $B(0)=0$.

For the low-pH experiment, the kinetics are more complicated owing to the distribution of hostguest binding in solution. We use a first-order kinetic model with rate matrix $\underline{K}$ :

$$
\underline{K}=\left(\begin{array}{cccc}
-k_{A \rightarrow B} & 0 & 0 & 0 \\
k_{A \rightarrow B} & -k_{B \rightarrow G S} & 0 & 0 \\
0 & 0 & -k_{C \rightarrow D} & 0 \\
0 & 0 & k_{C \rightarrow D} & 0
\end{array}\right)
$$

with $A(0)=1 ; B(0)=0, C(1)=0$, and $D(0)=0$.

The MATLAB program solves numerically the differential equations through matrix methods, ${ }^{2}$ then convolutes the solutions with a Gaussian instrument response function with width $w$ (FWHM), before employing a least-squares fitting routine using a Levenberg-Marquardt or Simplex method to find the parameters which result in matches to the kinetic data.

Once the fit parameters are established, they are fed directly into the differential equations, which were solved for the populations of the states in the model-i.e., $A(t), B(t), C(t)$, and $D(t)$. Finally, 
the raw data matrix (with all the raw data) is deconvoluted with the populations as functions of time to produce the spectra associated with each species.

\section{Section B. Synthetic Protocols}

\section{1) Complexation of ExBox ${ }^{4+}$ and DPP in $\mathrm{H}_{2} \mathrm{O}$}

The synthesis of $\mathbf{E x B o x} \cdot 4 \mathrm{PF}_{6}$ was carried out according to a literature procedure ${ }^{3}$. ExBox$\bullet 4 C l$ was precipitated by dissolution of $\mathbf{E x B o x} \cdot 4 \mathrm{PF}_{6}$ in $\mathrm{MeCN}$, followed by the addition of ${ }^{\mathrm{n}} \mathrm{Bu}_{4} \mathrm{NCl}$. The precipitate was collected, and excess of ${ }^{n} \mathrm{Bu}_{4} \mathrm{NCl}$ was removed by multiple $\mathrm{H}_{2} \mathrm{O}$ washing to yield pure ExBox•4Cl.

Solid 5,15-diphenylporphyrin $(0.46 \mathrm{mg}, 1 \mathrm{mmol})$ was dissolved in $\mathrm{Me}_{2} \mathrm{CO}(1 \mathrm{mM}, 1 \mathrm{~mL})$ and added to a solution of $\mathbf{E x B o x} \cdot 4 \mathrm{Cl}$ in $\mathrm{H}_{2} \mathrm{O}(1.0 \mathrm{mM}, 1 \mathrm{~mL})$. The mixture was sonicated for $5 \mathrm{mins}$, $\mathrm{Me}_{2} \mathrm{CO}$ was removed in vacuum, and the aqueous solution was passed through a $0.45 \mu \mathrm{m}$ filter to yield $\mathbf{E x B o x}^{4+} \supset \mathbf{D P P}$ complex in $\mathrm{H}_{2} \mathrm{O}$.

\section{Section C. NMR Spectroscopy}

a) ${ }^{1} \mathrm{H}$ NMR Spectrum of ExBox$\cdot 4 \mathrm{PF}_{6}$ in $\left(\mathrm{CD}_{3}\right)_{2} \mathrm{CO}$

\begin{tabular}{|c|c|c|}
\hline $\begin{array}{l}m \\
0 \\
m \\
m\end{array}$ & $\begin{array}{l}\hat{n} \text { ब } \\
\text { ñ }\end{array}$ & $\stackrel{m}{\stackrel{m}{c}}$ \\
\hline बi & $\infty \infty^{\circ}$ & $\infty$ \\
\hline
\end{tabular}

今్
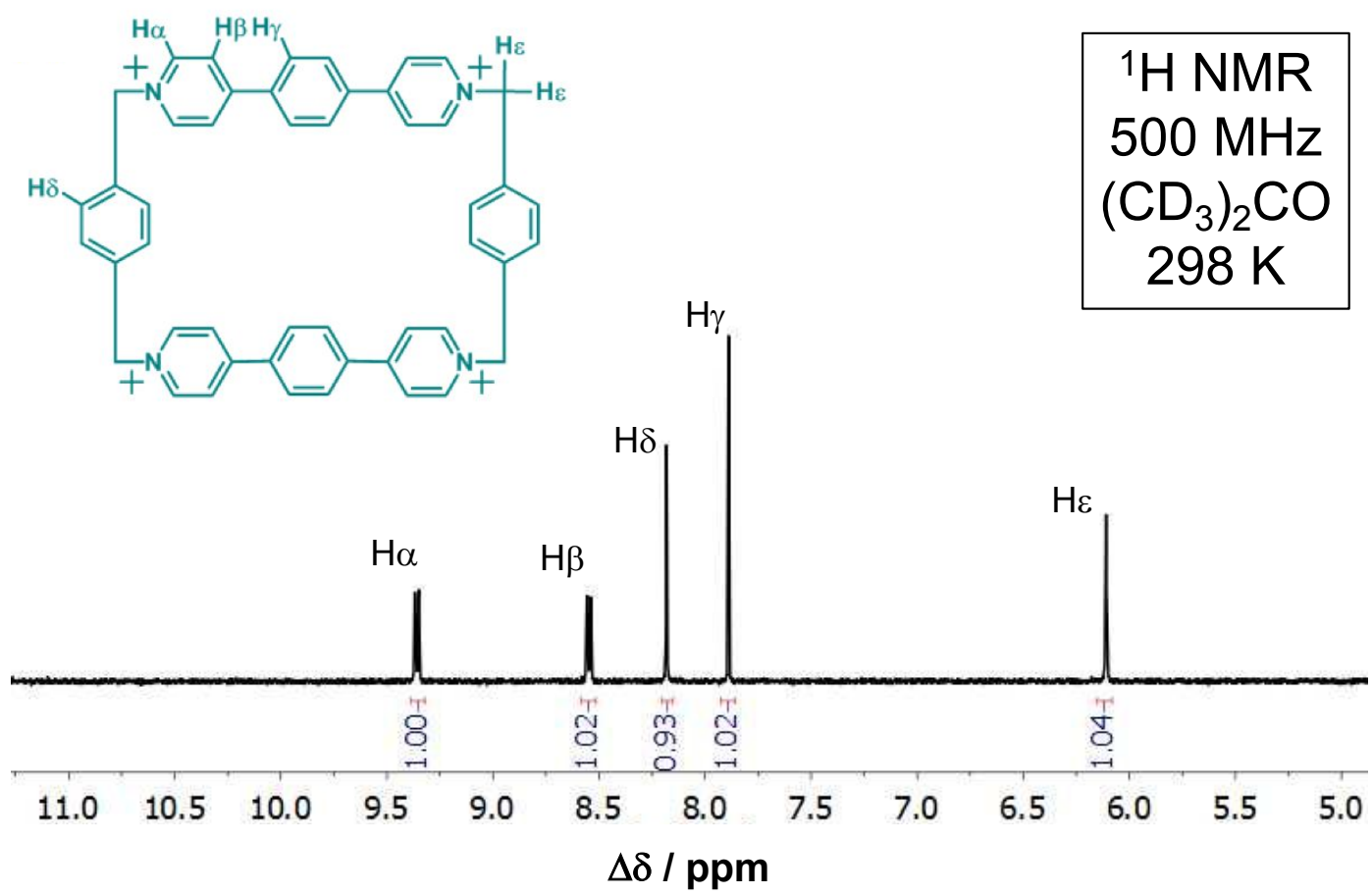
Supplementary Figure 1. Annotated ${ }^{1} \mathrm{H}$ NMR spectrum $\left(500 \mathrm{MHz},\left(\mathrm{CD}_{3}\right)_{2} \mathrm{CO}, 298 \mathrm{~K}\right)$ of ExBox$\bullet 4 \mathrm{PF}_{6}$

b) ${ }^{1} \mathrm{H} N M R$ Spectra of ExBox ${ }^{4+} \mathrm{DPP}, \mathrm{ExBox} \bullet 4 \mathrm{PF}_{6}$ and DPP in $\left(\mathrm{CD}_{3}\right)_{2} \mathrm{CO}$
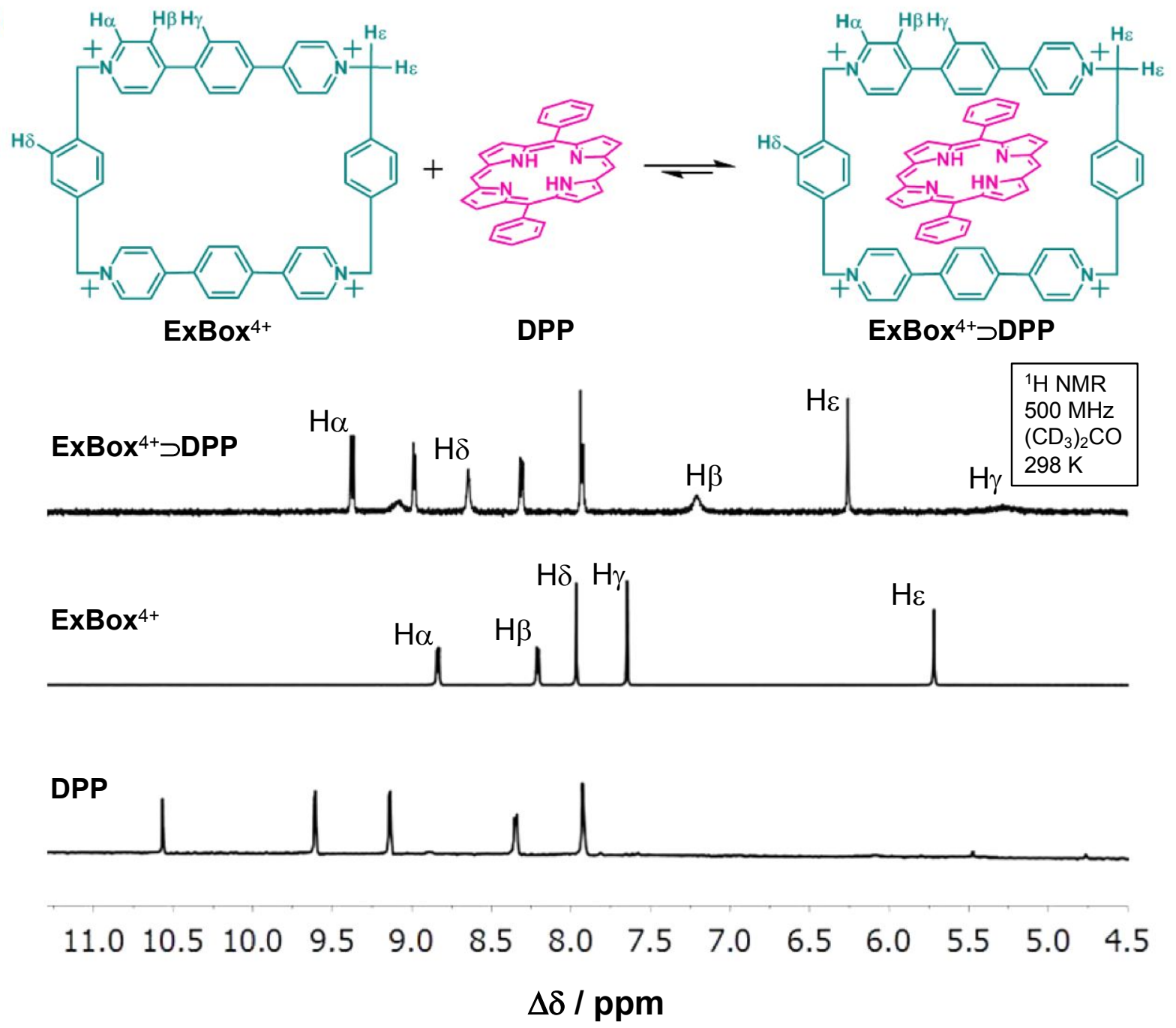

Supplementary Figure 2. Annotated ${ }^{1} \mathrm{H}$ NMR spectra $\left(500 \mathrm{MHz},\left(\mathrm{CD}_{3}\right)_{2} \mathrm{CO}, 298 \mathrm{~K}\right)$ of $\mathbf{E x B o x}^{4+} \supset \mathbf{D P P}, \mathbf{E x B o x} \bullet 4 \mathrm{PF}_{6}$ and DPP

c) ${ }^{1} \mathrm{H}$ NMR Titration of ExBox$\bullet 4 \mathrm{PF}_{6}$ and $\mathrm{DPP}$ in $\left(\mathrm{CD}_{3}\right)_{2} \mathrm{CO}$ and calculation of binding constant

Stock solutions of $\mathbf{E x B o x}{ }^{4+}(0.4 \mathrm{mM})$ and DPP $(5.4 \mathrm{mM})$ dissolved in $\left(\mathrm{CD}_{3}\right)_{2} \mathrm{CO}$ were prepared. A $500 \mu \mathrm{L}$ aliquot of $\mathbf{E x B o x}{ }^{4+}$ was introduced into an NMR tube and titrated with the DPP solution. The NMR spectra were recorded after each addition of the DPP solution. The binding constant 
was obtained from a 1:1 binding model by utilizing the change in the chemical shift value of the probe $(*)$ peak. 


\section{Section D. Crystallographic Characterization}

All the crystallographic data for the (super)structure reported in this paper have been deposited in the Cambridge Crystallographic Data Centre (CCDC) and can be obtained free of charge via www.ccdc.cam.ac.uk/data_request/cif. CCDC deposition number 1903587.

a) Method: Single crystals of $\mathbf{E x B o x}{ }^{4+} \supset \mathbf{D P P}$ were grown by slow vapor diffusion of ${ }^{i} \operatorname{Pr}_{2} \mathrm{O}$ into a solution of $\mathbf{E x B o x}^{4+}$ and DPP in $\mathrm{Me}_{2} \mathrm{CO} / \mathrm{MeCN}$ (1:1) over the course of 1 week. A suitable crystal was selected, and the crystal was mounted on a MITIGEN holder in Paratone oil on a Bruker Kappa APEX CCD area detector diffractometer. The crystal was kept at $100 \mathrm{~K}$ during data collection. Using ${ }^{4}$ Olex2, the structure was solved with the ShelXT ${ }^{5}$ structure solution program using Direct Methods and refined with the ShelXL ${ }^{6}$ refinement package using Least Squares minimization.

b) Crystal Data for $\mathrm{C}_{87} \mathrm{H}_{74} \mathrm{~F}_{24} \mathrm{~N}_{10} \mathrm{OP}_{4}$, Monoclinic, space group P2/c (no. 13), $a=31.4603(19), b$ $=15.0357(9), c=20.4599(10) \AA, \beta=104.239(4)^{\circ}, V=9380.8(9) \AA^{3}, Z=4, T=100.01 \mathrm{~K}, \mu(\mathrm{CuK} \alpha)$ $=1.594 \mathrm{~mm}^{-1}, D_{\text {calc }}=1.314 \mathrm{~g} / \mathrm{mm}^{3}, 49019$ reflections measured $(2.898 \leq 2 \Theta \leq 130.472), 15796$ unique $\left(R_{\text {int }}=0.0379, \mathrm{R}_{\text {sigma }}=0.0422\right)$ which were used in all calculations. The final $R_{1}$ was 0.0925 $(I>2 \sigma(I))$ and $w R_{2}$ was 0.2865 (all data).

c) Refinement Details. Distance restraints were imposed on the disordered $\mathrm{PF}_{6}$ anions. The enhanced rigid-bond restraint (SHELX keyword RIGU) was applied globally. ${ }^{4}$ The solvent masking procedure as implemented in Olex2 was used to remove the electronic contribution of heavily disordered solvent molecules from the refinement. As the exact solvent content is not known, only the atoms used in the refinement model are reported in the formula here. Total solvent accessible volume $/$ cell $=1575.4 \AA^{3}[16.8 \%]$ Total electron count $/$ cell $=377.8$. 
(a)

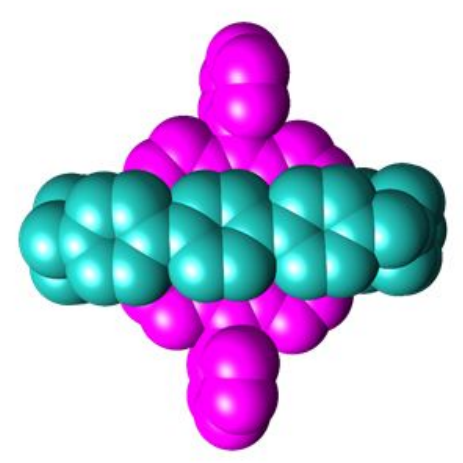

Side view

(c)

(e)

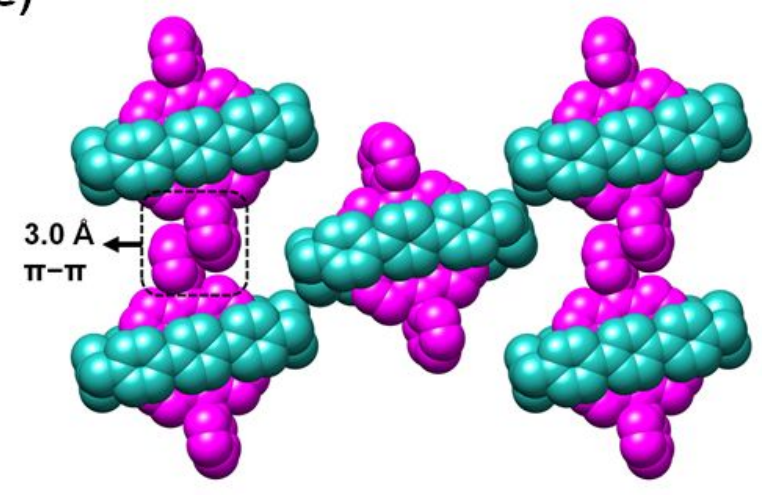

(b)
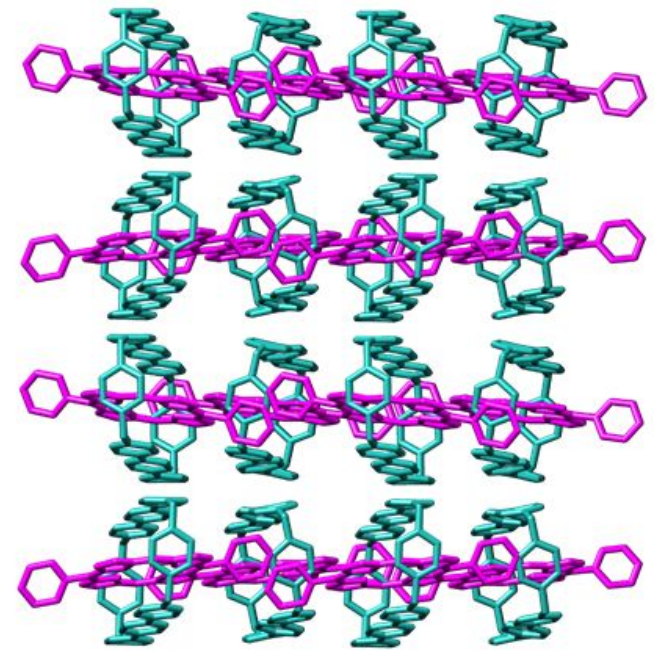

(d)

Viewed along the a-axis

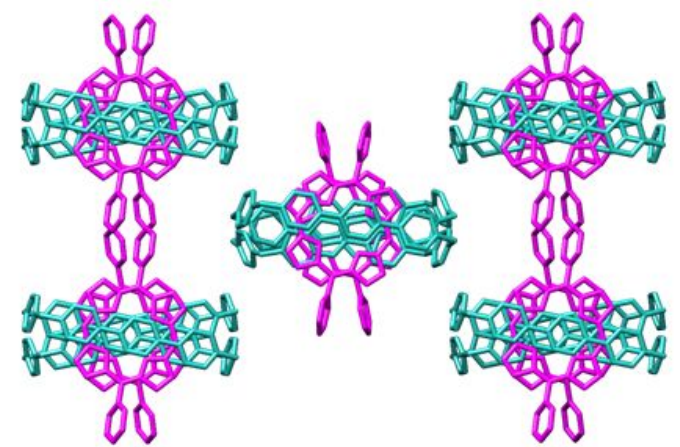

Viewed along the $c$-axis

(f)

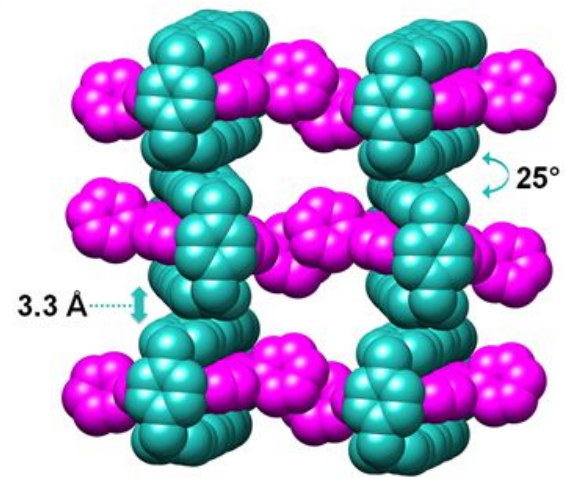

Supplementary Figure 3. Solid-state (super)structures of the $\operatorname{ExBox}^{4+} \supset$ DPP obtained from single-crystal X-ray crystallography. (a) Space-filling representation of $\mathbf{E x B o x}^{4+} \supset \mathbf{D P P}$ complex. (b-d) Tubular representations of $\mathbf{E x B o x}^{4+} \supset \mathbf{D P P}$ superstructures viewed along $a, b$, and $c$-axis respectively. (e) Space-filling representations showing intermolecular $\pi-\pi$ interactions between the two ExBIPY ${ }^{2+}$ sides of ExBox ${ }^{4+} \supset \mathbf{D P P}$ complexes. (f) Space-filling representation showing an alternate offset arrangement of the neighboring $\mathbf{E x B o x}^{4+} \supset$ DPP complexes. 
Section E. Transient Absorption Spectroscopy

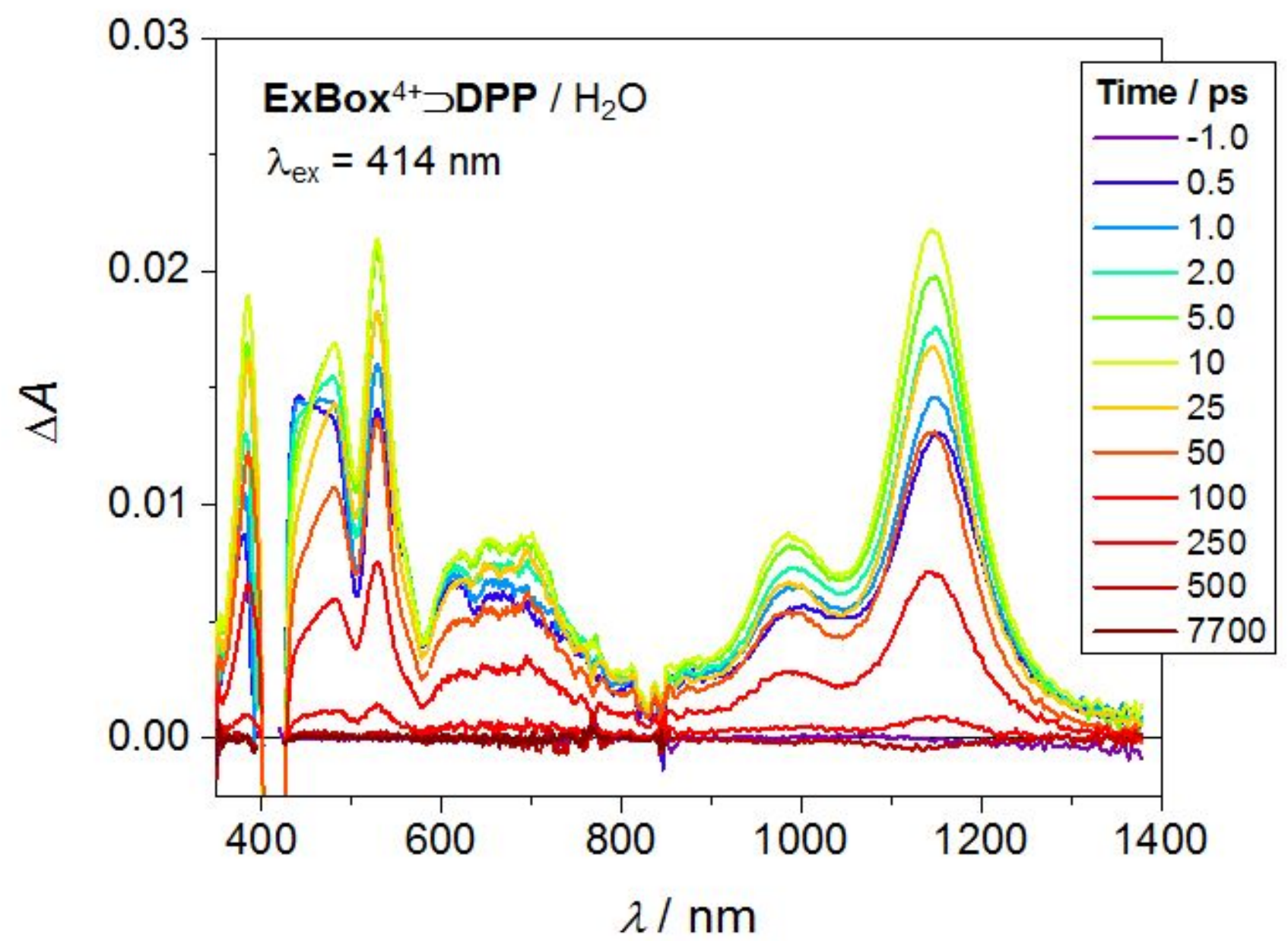

Supplementary Figure 4. Femtosecond transient absorption spectra of $\mathbf{E x B o x}{ }^{4+} \supset \mathbf{D P P}$ in $\mathrm{H}_{2} \mathrm{O}$ excited at $\lambda_{\text {ex }}=414 \mathrm{~nm}$ 
(a)

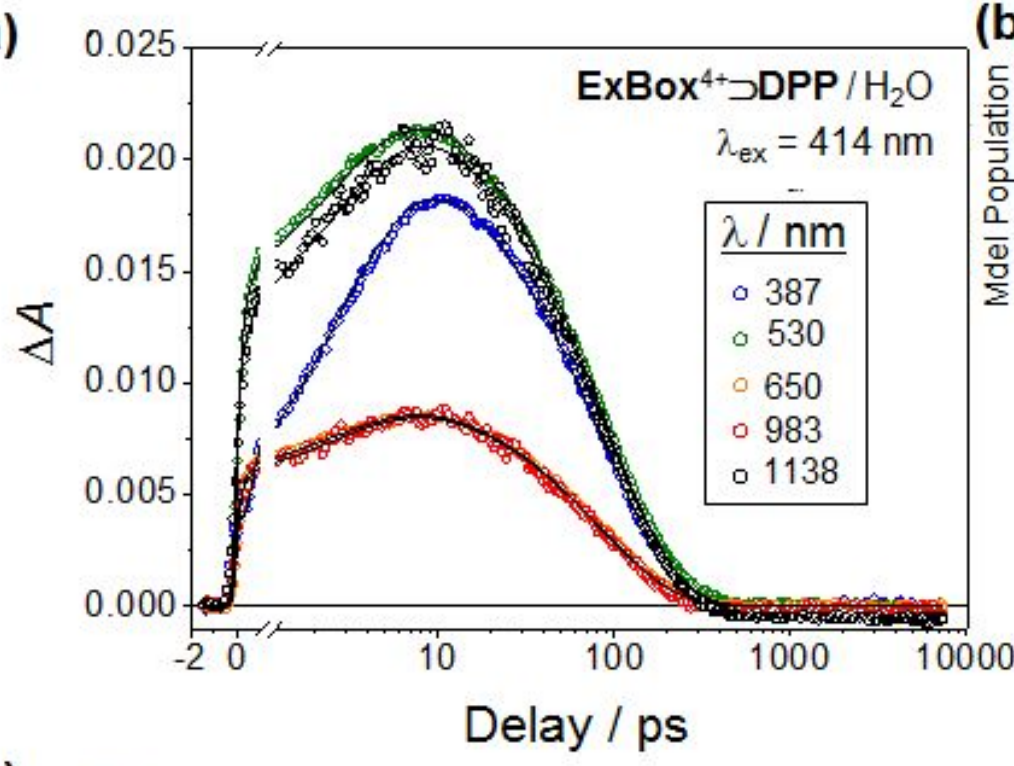

(b)

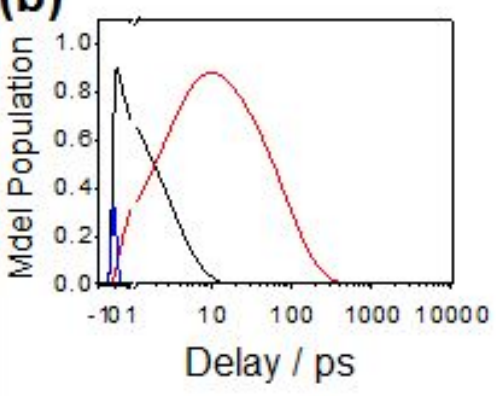

$$
\begin{aligned}
\underline{K} & =\left[\begin{array}{cc}
-k_{1} & 0 \\
k_{1} & -k_{2}
\end{array}\right] \\
\tau_{1} & =2.9 \pm 0.3 \mathrm{ps} \\
\tau_{2} & =81.8 \pm 0.3 \mathrm{ps}
\end{aligned}
$$

(c)

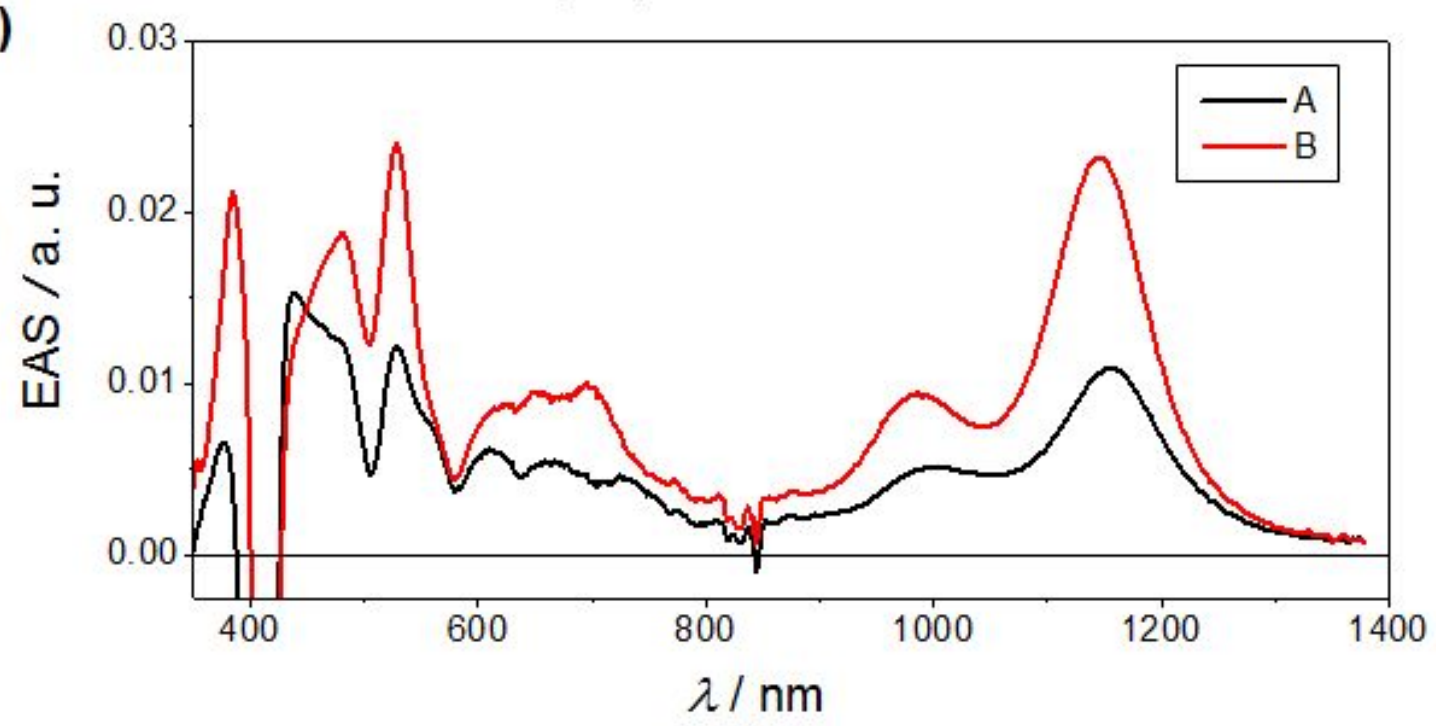

Supplementary Figure 5. Kinetic analysis of femtosecond transient data for $\operatorname{ExBox}^{4+} \supset \mathbf{D P P}$ in $\mathrm{H}_{2} \mathrm{O}$ excited at $\lambda_{\mathrm{ex}}=414 \mathrm{~nm}$. (a) Kinetic traces at selected wavelengths with fits (black lines). (b) Model populations for the fit to a two-state sequential kinetic model with rate matrix shown. (c) Evolution-associated spectra (EAS) for the two states in the model. State A represents the locally excited ${ }^{1 *}$ DPP state and some degree of charge-separated state formed within the instrument response, and state B is the fully charge-separated state. 


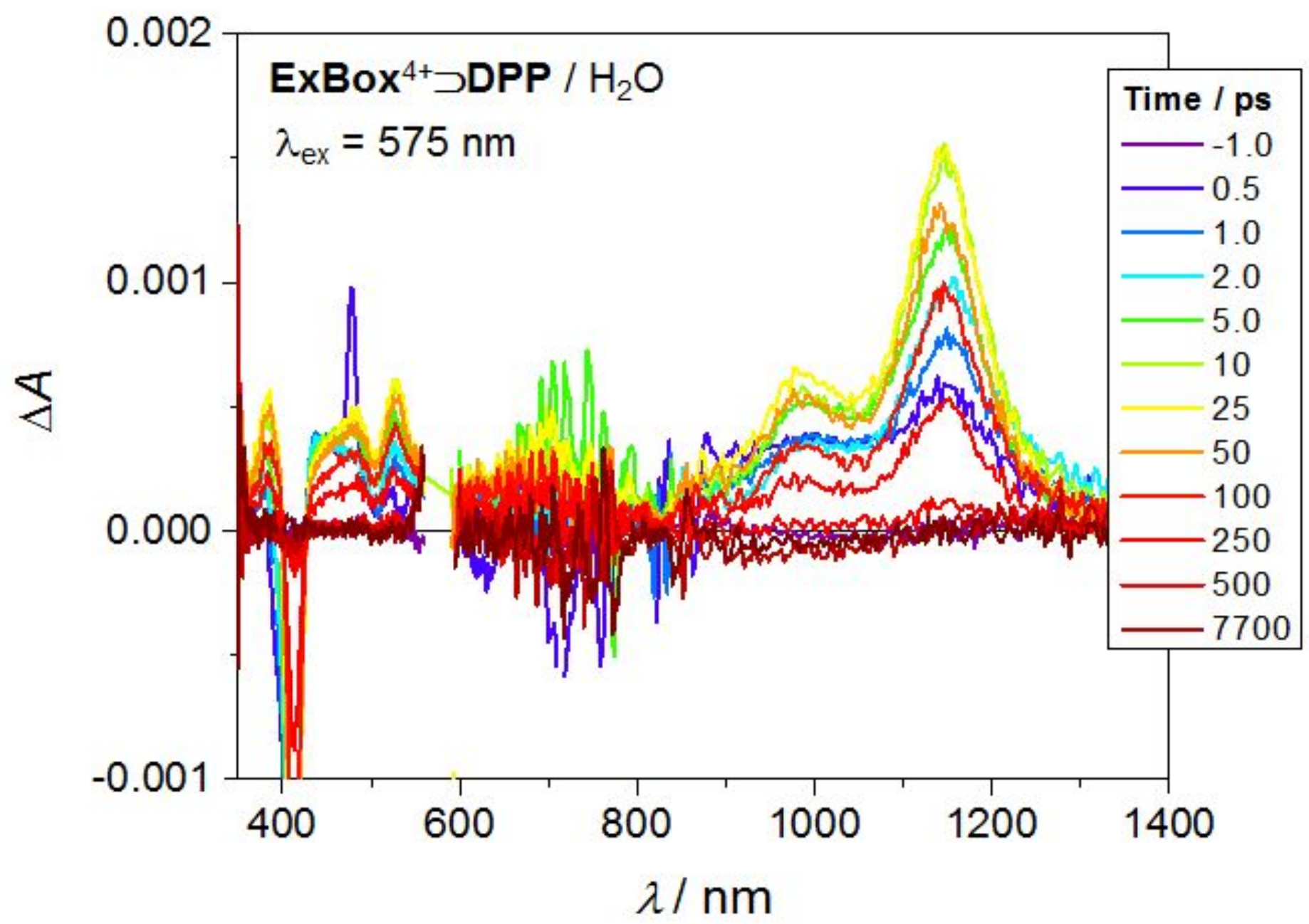

Supplementary Figure 6. Femtosecond transient absorption spectra of $\mathbf{E x B o x}{ }^{4+} \supset \mathbf{D P P}$ in $\mathrm{H}_{2} \mathrm{O}$ excited at $\lambda_{\text {ex }}=575 \mathrm{~nm}$ 
(a)

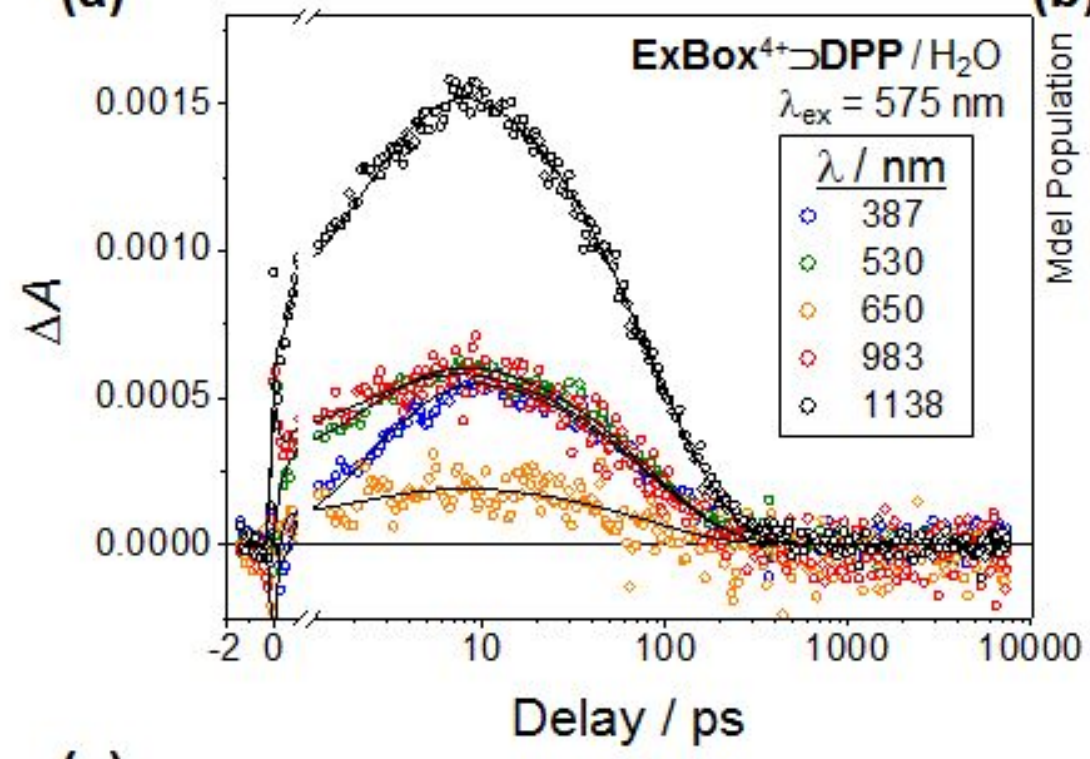

(b)

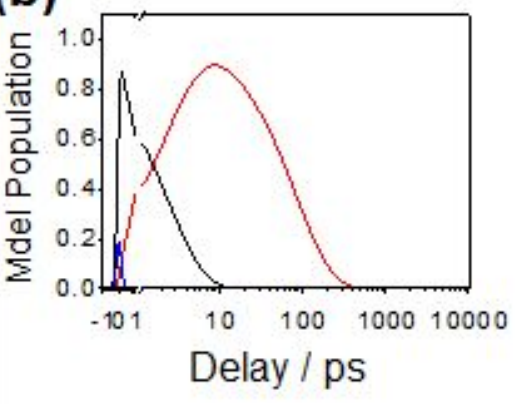

$$
\begin{aligned}
\underline{K} & =\left[\begin{array}{cc}
-k_{1} & 0 \\
k_{1} & -k_{2}
\end{array}\right] \\
\tau_{1} & =2.5 \pm 0.3 \mathrm{ps} \\
\tau_{2} & =83.6 \pm 0.3 \mathrm{ps}
\end{aligned}
$$

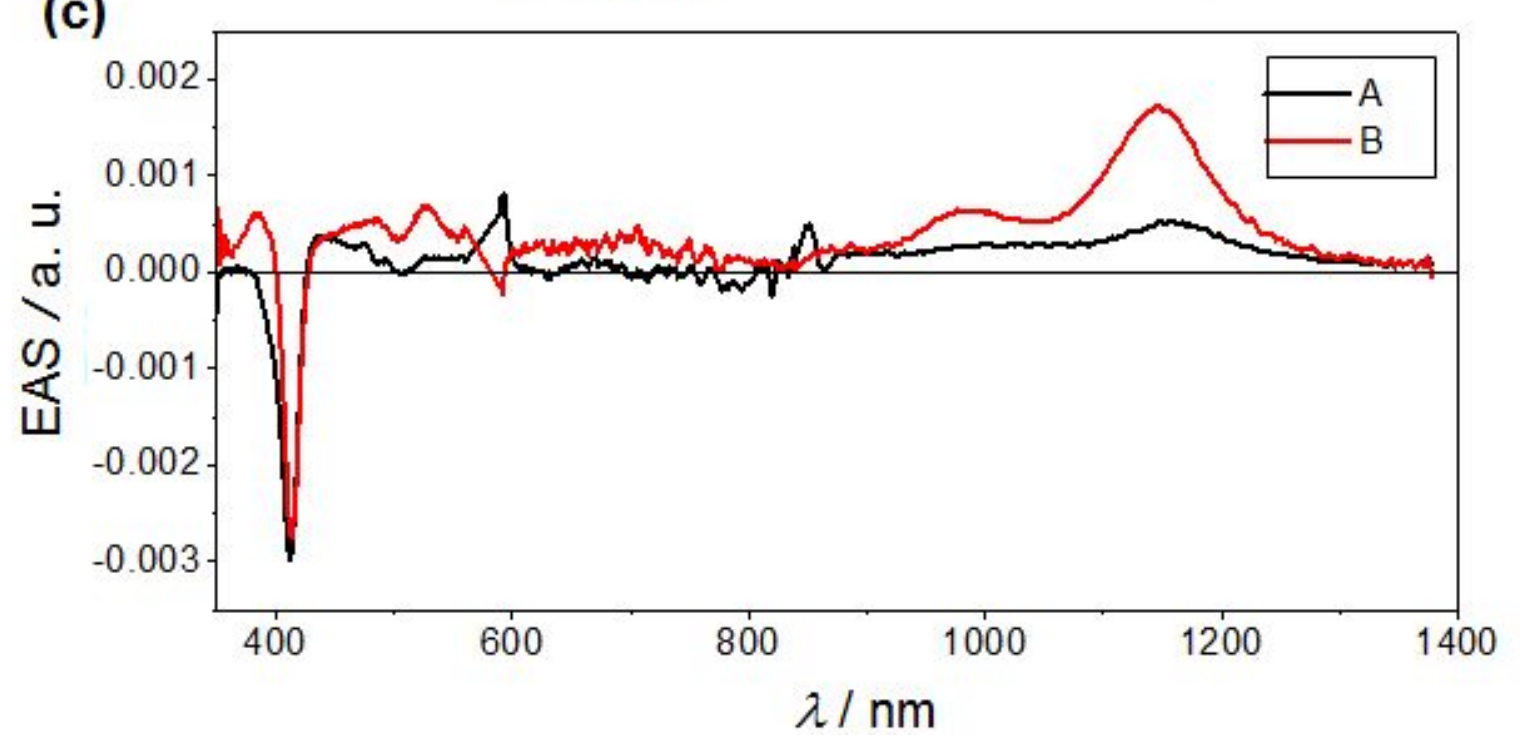

Supplementary Figure 7. Kinetic analysis of femtosecond transient data for $\mathbf{E x B o x}{ }^{4+} \supset \mathbf{D P P}$ in $\mathrm{H}_{2} \mathrm{O}$ excited at $\lambda_{\mathrm{ex}}=575 \mathrm{~nm}$. (a) Kinetic traces at selected wavelengths with fits (black lines). (b) Model populations for the fit to a two-state sequential kinetic model with rate matrix shown. (c) Evolution-associated spectra (EAS) for the two states in the model. State A represents the locally excited ${ }^{1 *}$ DPP state and some degree of charge-separated state formed within the instrument response, and state $\mathrm{B}$ is the fully charge-separated state. 


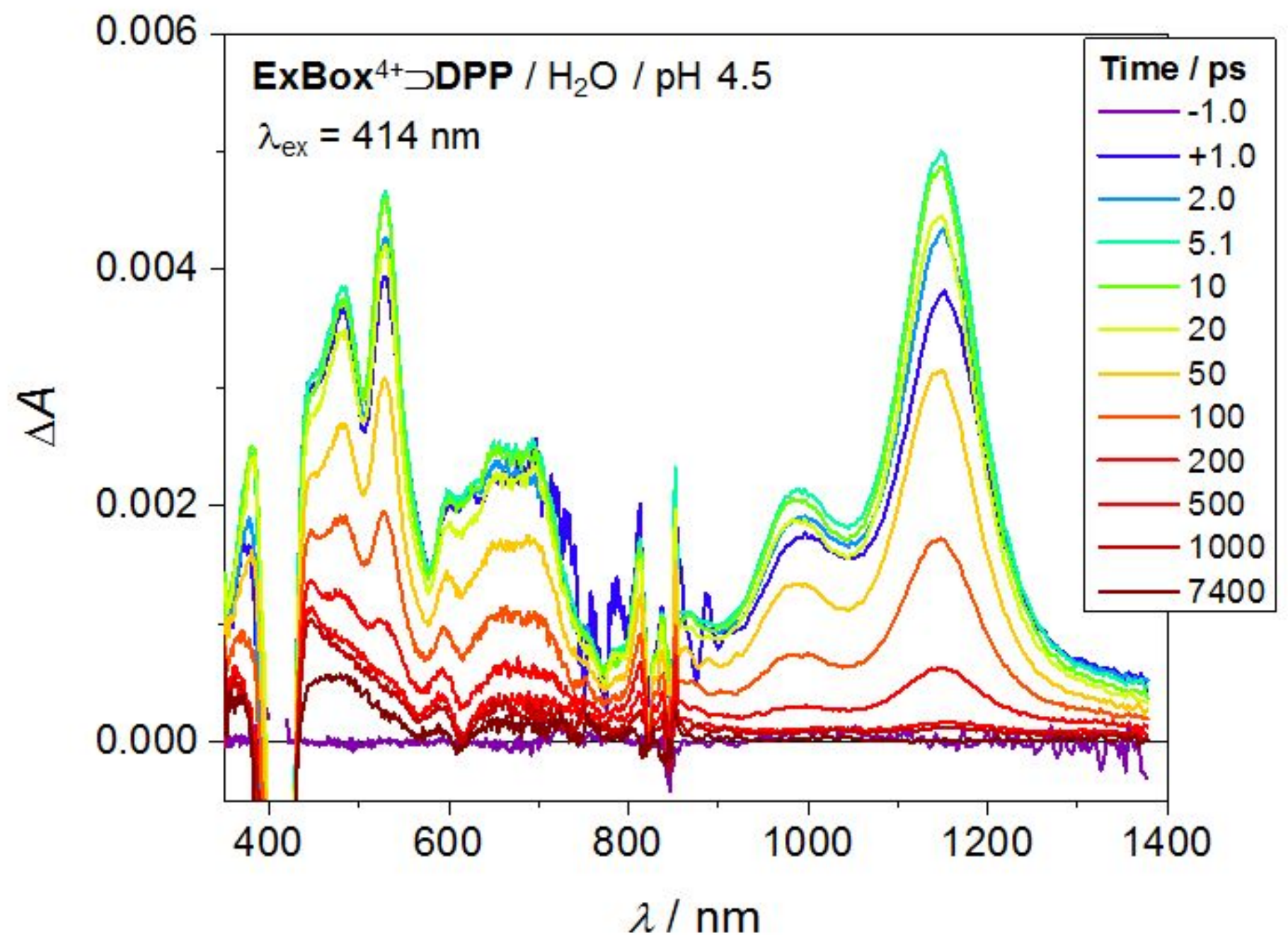

Supplementary Figure 8. Femtosecond transient absorption spectra of $\mathbf{E x B o x}{ }^{4+} \supset \mathbf{D P P}$ in $\mathrm{H}_{2} \mathrm{O}$ with added $\mathrm{HCl}$ (pH adjusted to 4.5) excited at $\lambda_{\text {ex }}=414 \mathrm{~nm}$ 

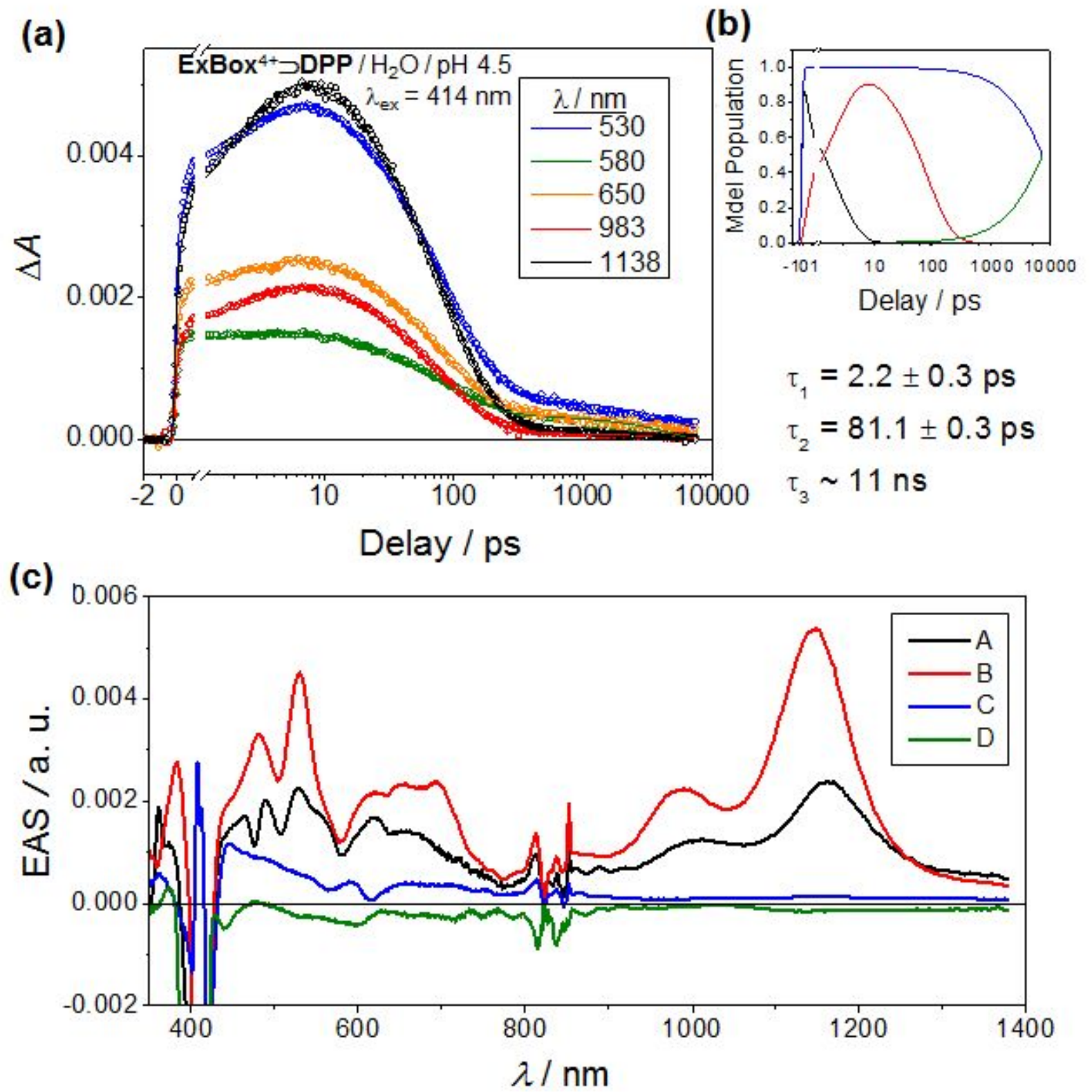

Supplementary Figure 9. Kinetic analysis of femtosecond transient data for $\mathbf{E x B o x}{ }^{4+} \supset \mathbf{D P P}$ in $\mathrm{H}_{2} \mathrm{O}$ with added $\mathrm{HCl}$, excited at $\lambda_{\mathrm{ex}}=414 \mathrm{~nm}$. (a) Kinetic traces at selected wavelengths with fits (black lines). (b) Model populations for the fit to a two-state sequential kinetic model with time constants shown. (c) Evolution-associated spectra (EAS) for the two states in the model. State A represents the locally excited ${ }^{1 *}$ DPP state and some degree of charge-separated state formed within the instrument response, and state B is the fully charge-separated state; the other states C and D represent the ${ }^{1 *} \mathbf{D} \mathbf{P} \mathbf{P H}_{2}{ }^{2+}$ and ${ }^{3 *} \mathbf{D P P H} \mathbf{H}^{2+}$ populations, respectively, of the free $\mathbf{D P P H} \mathbf{2}^{2+}$ in solution. 

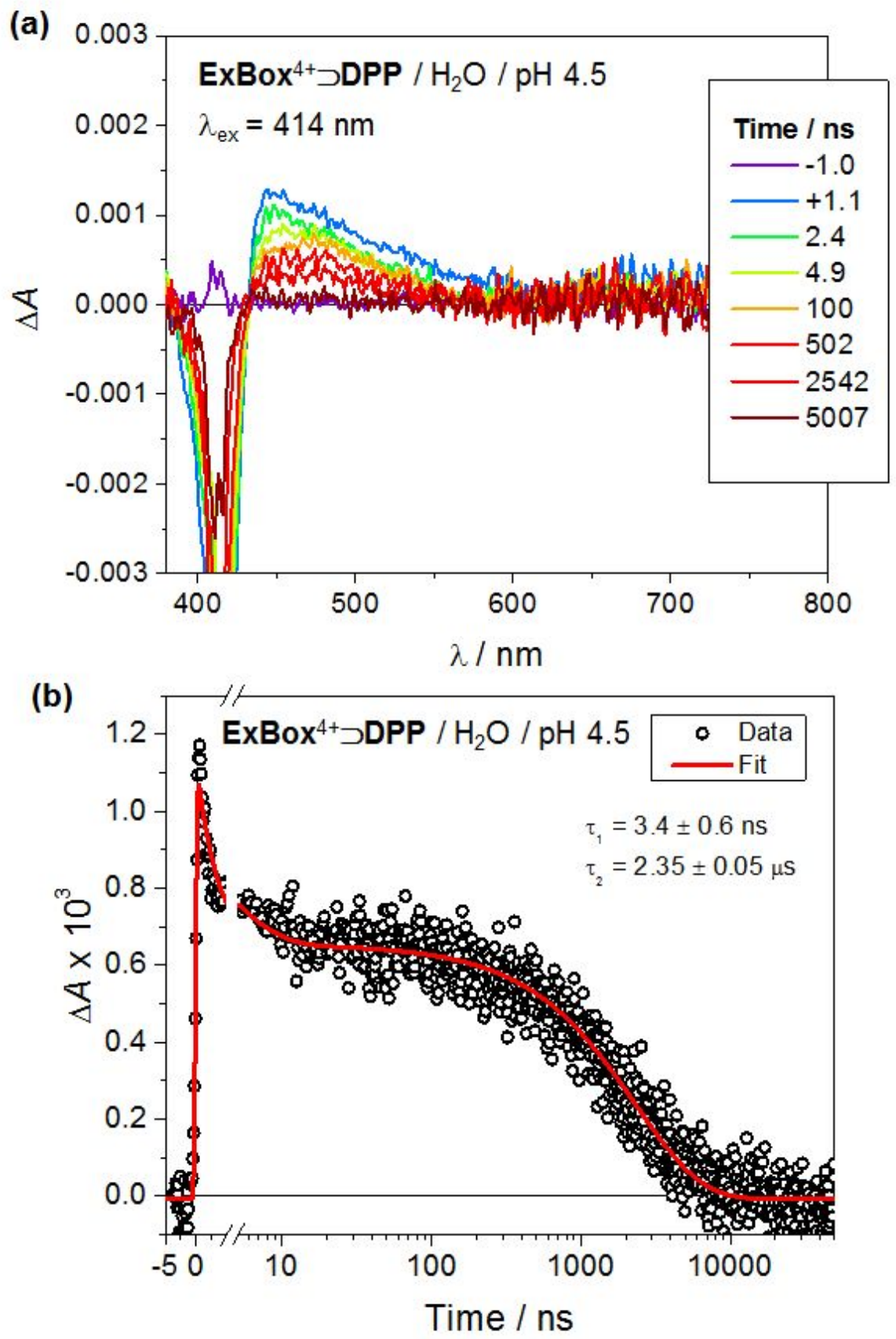

Supplementary Figure 10. (a) Nanosecond TA (nsTA) spectra of $\mathbf{E x B o x}{ }^{4+} \supset \mathbf{D P P}$ in $\mathrm{H}_{2} \mathrm{O}$ with added $\mathrm{HCl}$ excited at $\lambda_{\text {ex }}=414 \mathrm{~nm}$. (b) Biexponential fit to nsTA data at $475 \mathrm{~nm}$. The first time constant represents the intersystem crossing time from the $S_{1}$ to $T_{1}$ states, while the second time constant approximates the decay of the triplet state in aerated, room temperature solution. The disagreement with the $\sim 3$ ns intersystem crossing time determined with nsTA and the longer time approximated in the fsTA is a result of the limited time range of the fsTA measurement and the complexity of the kinetics owing to the heterogeneity of the sample. 


\section{Section F. Detection of Singlet Oxygen}

Singlet oxygen $\left({ }^{1} \mathrm{O}_{2}\right)$ quantum-yield measurements were conducted by a chemical method. The disodium salt of 9.10-anthracenedipropionic acid (ADPA) was used as a singlet oxygen detection reagent with methylene blue used as a reference $\left(\Phi_{\mathrm{r}}=0.53\right)$. In this experiment, ADPA $(0.1 \mu \mathrm{mol})$ was added to the $\mathrm{H}_{2} \mathrm{O}$ solution of $\mathbf{E x B o x}{ }^{4+} \supset \mathbf{D P P}(20 \mu \mathrm{M})$ with a laser irradiation $(630 \mathrm{~nm}, 50$ $\mathrm{mW}$ ) for $20 \mathrm{~min}$, followed by quantification of the ${ }^{1} \mathrm{O}_{2}$ by the absorbance intensity change (UV3600 Shimadzu spectrophotometer) at $380 \mathrm{~nm}$ of ADPA. The calculation of the ${ }^{1} \mathrm{O}_{2}$ quantum yield is based on the equality $\Phi \Delta=\left(\Delta A_{\mathrm{s}} / \Delta A_{\mathrm{r}}\right) \Phi_{\mathrm{r}}$ where $\Delta A_{\mathrm{s}}$ is the absorption change of detected photosensitizer, $\Delta \mathrm{A}_{\mathrm{r}}$ is the absorption change of referenced photosensitizer and $\Phi_{\mathrm{r}}$ is the quantum yield of singlet oxygen of referenced photosensitizer (methylene blue). No change of absorption $\left(\triangle A_{\mathrm{s}} \sim 0\right)$ confirmed that ${ }^{1} \mathrm{O}_{2}$ was not generated during the experiment.

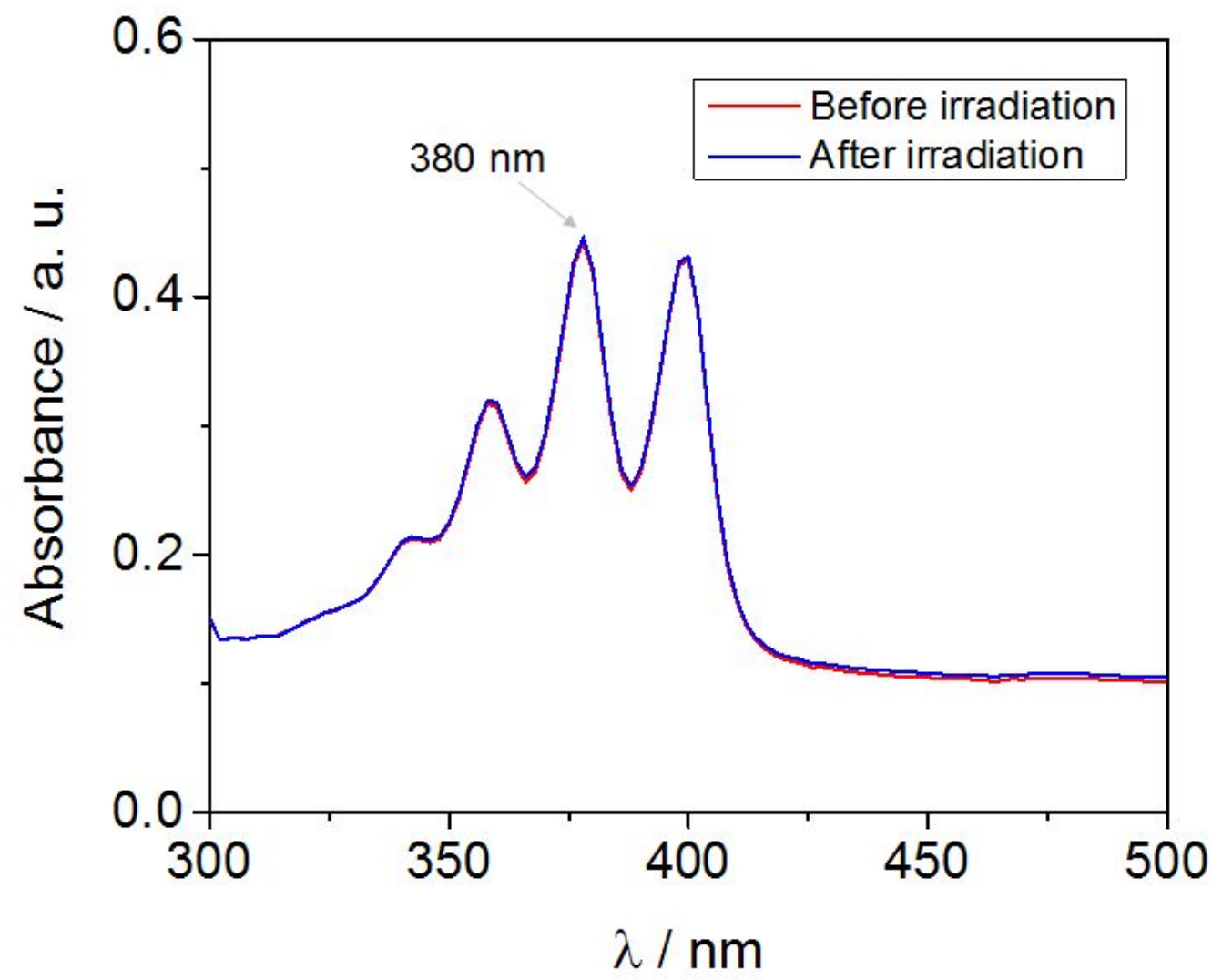

Supplementary Figure 11. UV-Vis spectra of a mixed solution of ADPA and $\operatorname{ExBox}^{4+} \supset$ DPP showing no change of absorbance intensity at $380 \mathrm{~nm}$ before and after irradiation in $\mathrm{H}_{2} \mathrm{O}$. 
ADPA $(0.1 \mu \mathrm{mol})$ was added to the $\mathrm{H}_{2} \mathrm{O}$ solution of $\mathbf{D P P H} \mathbf{H}^{2+}(20 \mu \mathrm{M})$ with a laser irradiation $(630 \mathrm{~nm}, 50 \mathrm{~mW})$ for $20 \mathrm{~min}$, followed by quantification of the ${ }^{1} \mathrm{O}_{2}$ by the absorbance intensity change (UV-3600 Shimadzu spectrophotometer) at $380 \mathrm{~nm}$ of ADPA. The calculation of the ${ }^{1} \mathrm{O}_{2}$ quantum yield is based on the equality $\Phi \Delta=\left(\Delta A_{\mathrm{s}} / \Delta A_{\mathrm{r}}\right) \Phi_{\mathrm{r}}$ where $\Delta A_{\mathrm{s}}$ is the absorption change of detected photosensitizer, $\Delta \mathrm{A}_{\mathrm{r}}$ is the absorption change of referenced photosensitizer and $\Phi_{\mathrm{r}}$ is the quantum yield of singlet oxygen of referenced photosensitizer (methylene blue). Measured ${ }^{1} \mathrm{O}_{2}$ quantum yield of $\mathbf{D P P H _ { 2 }}{ }^{2+}$ is 0.38 in $\mathrm{H}_{2} \mathrm{O}$.

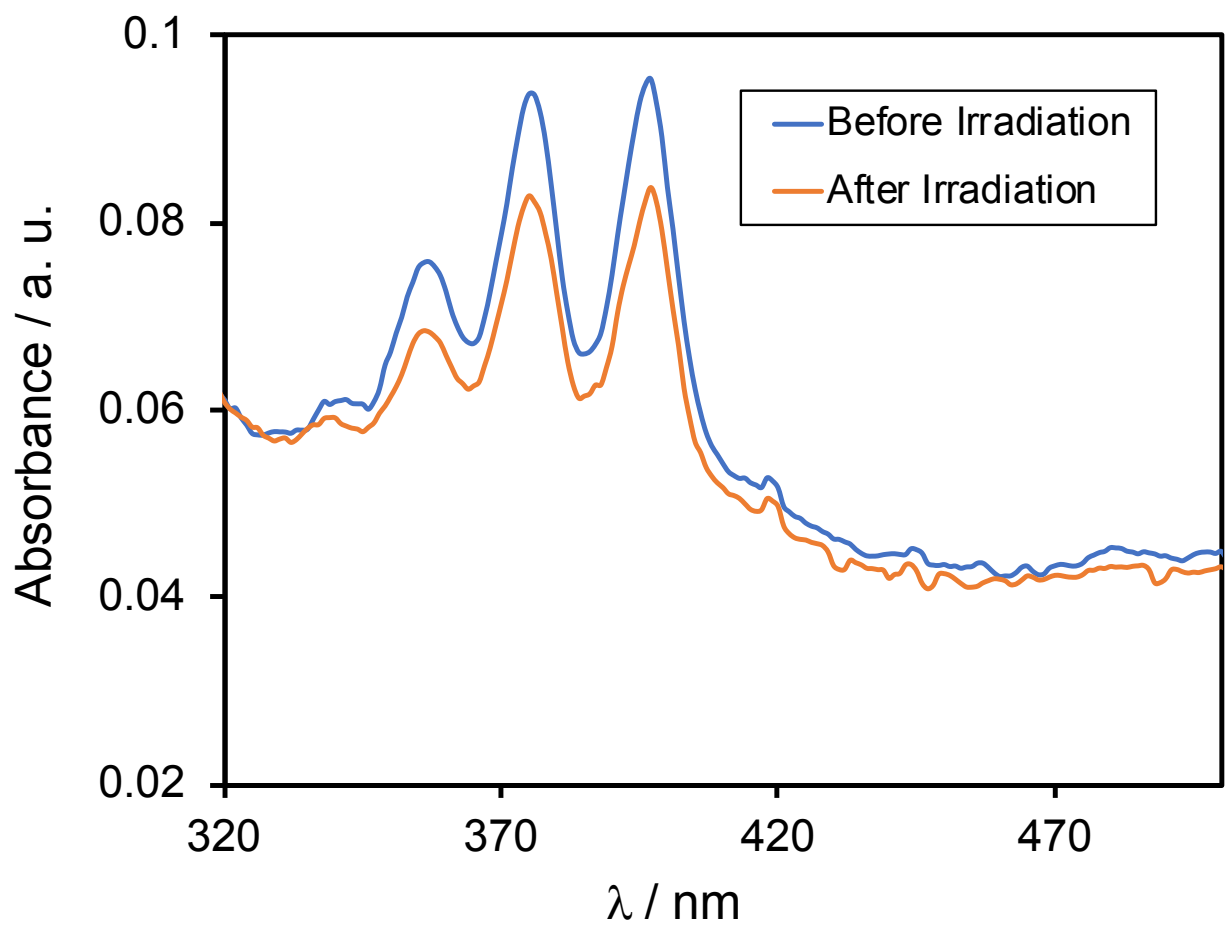

Supplementary Figure 12. UV-Vis spectra of a mixed solution of ADPA and $\mathbf{D P P H}_{2}{ }^{2+}$ showing the decrease of absorbance intensity at $380 \mathrm{~nm}$ before and after irradiation in $\mathrm{H}_{2} \mathrm{O}$.

ADPA $(0.1 \mu \mathrm{mol})$ was added to the acetone solution of DPP $(20 \mu \mathrm{M})$ with a laser irradiation (630 $\mathrm{nm}, 50 \mathrm{~mW}$ ) for $20 \mathrm{~min}$, followed by quantification of the ${ }^{1} \mathrm{O}_{2}$ by the absorbance intensity change (UV-3600 Shimadzu spectrophotometer) at $380 \mathrm{~nm}$ of ADPA. The calculation of the ${ }^{1} \mathrm{O}_{2}$ quantum yield is based on the equality $\Phi \Delta=\left(\Delta A_{\mathrm{s}} / \Delta A_{\mathrm{r}}\right) \Phi_{\mathrm{r}}$ where $\Delta A_{\mathrm{s}}$ is the absorption change of detected photosensitizer, $\Delta \mathrm{A}_{\mathrm{r}}$ is the absorption change of referenced photosensitizer and $\Phi_{\mathrm{r}}$ is the quantum yield of singlet oxygen of referenced photosensitizer (methylene blue). Measured ${ }^{1} \mathrm{O}_{2}$ quantum yield of DPP is 0.43 in acetone. 


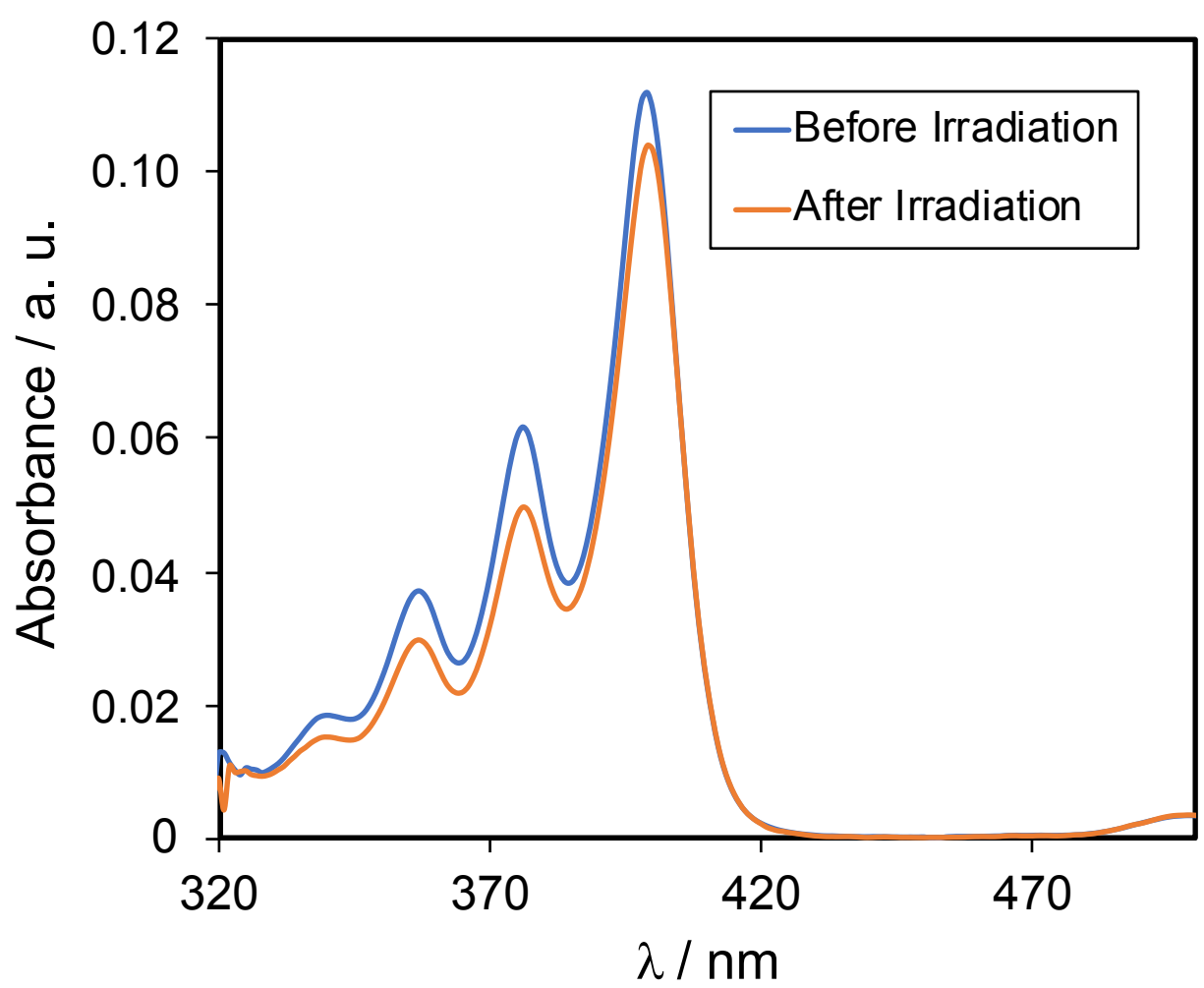

Supplementary Figure 13. UV-Vis spectra of a mixed solution of ADPA and DPP showing the decrease of absorbance intensity at $380 \mathrm{~nm}$ before and after irradiation in acetone.

\section{Section G. DFT Analysis}

The structures of DPP and $\mathbf{E x B o x}{ }^{4+} \supset \mathbf{D P P}$ were geometry optimized at the RB3LYP/6-31G*+ level and time-dependent DFT calculations were carried out on the optimized gas-phase geometries using Jaguar ${ }^{7}$, considering a total of 200 and 300 excited states for DPP and $\mathbf{E x B o x}^{4+} \supset \mathbf{D P P}$, respectively, to reach the upper end of the absorption spectrum (200 nm). Seventeen and twenty-six transitions were determined with oscillator strengths $>0.015$ for DPP and $\mathbf{E x B o x}{ }^{4+} \supset \mathbf{D P P}$, respectively, and these transitions are listed in Supplementary Tables 1 and 4. The frontier orbitals of DPP, relevant to the transition at 377.56 and $535.49 \mathrm{~nm}$, are presented in Supplementary Fig. 16. The frontier orbitals of $\mathbf{E x B o x}{ }^{4+} \supset \mathbf{D P P}$, relevant to the transition at 418.26 and $559.11 \mathrm{~nm}$, are presented in Supplementary Fig. 17. These transitions are not associated with a single dominant configuration amongst the excitation amplitudes and the major 
contributions to these transitions are given in Supplementary Tables 2 and 3 for DPP and in Supplementary Tables 5 and 6 for $\mathbf{E x B o x}^{4+} \supset \mathbf{D P P}$.
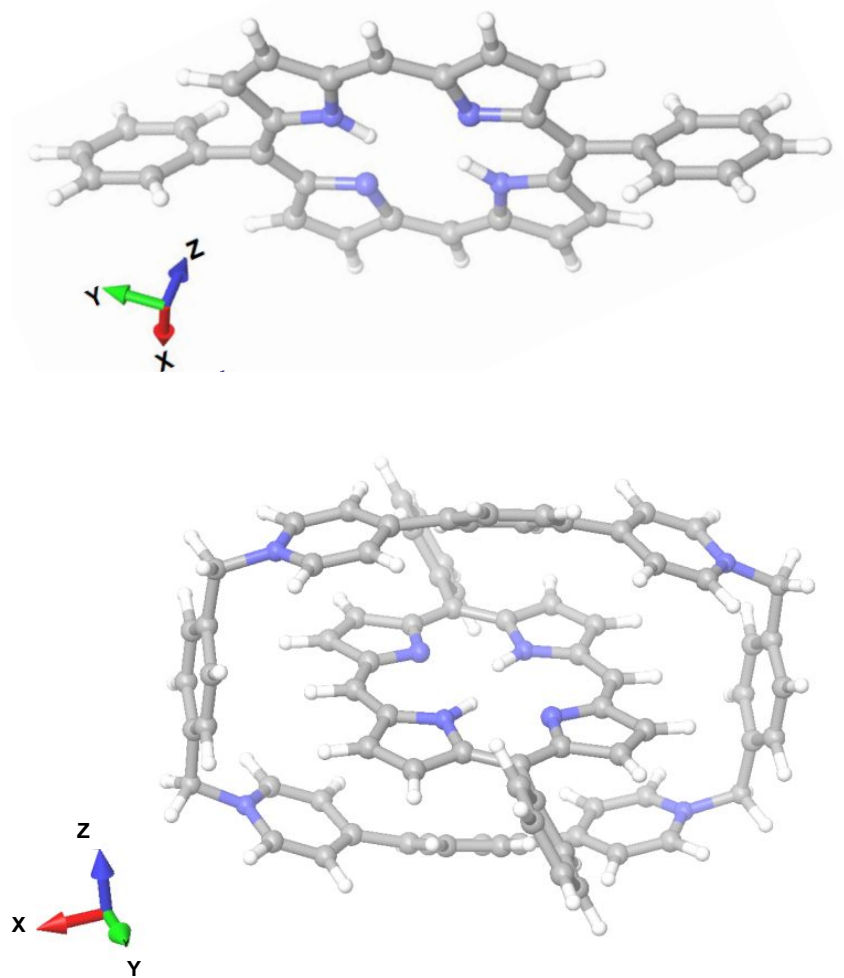

Supplementary Figure 14. Geometry-optimized structures of DPP (top) and $\mathbf{E x B o x}{ }^{4+} \supset \mathbf{D P P}$ (bottom).

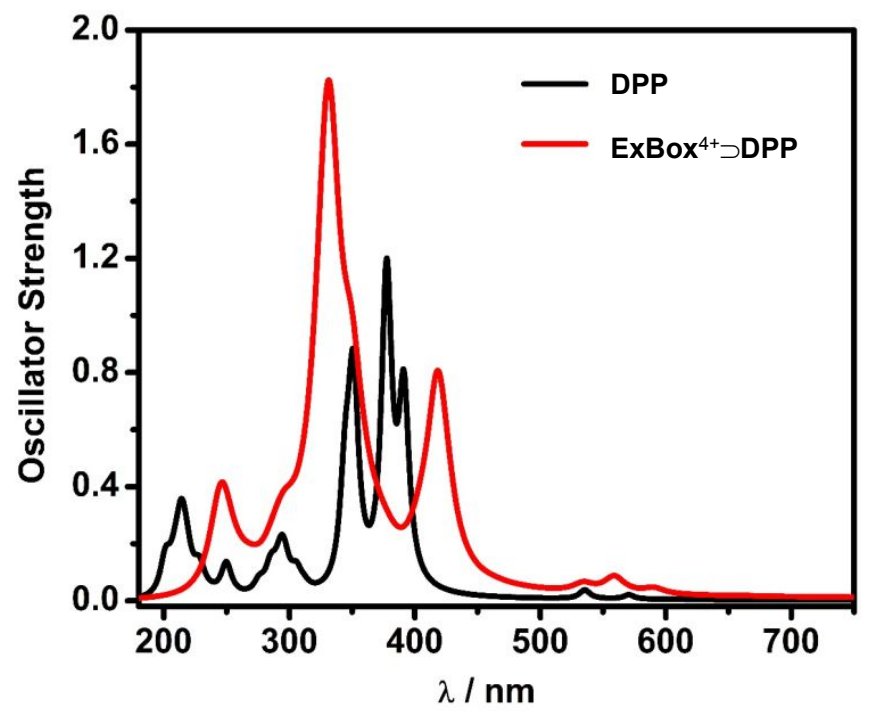

Supplementary Figure 15. Calculated UV-Vis spectra for DPP and $\mathbf{E x B o x}{ }^{4+} \supset \mathbf{D P P}$ (no scaling factor applied). 
Supplementary Table 1. RB3LYP/6-31G*+ computed transitions for DPP with oscillator strengths $(f)$ greater than 0.015 .

\begin{tabular}{cccc}
\hline Transition & Wavelength $(\mathrm{nm})$ & $f$ & Transition dipole moment (Debye) \\
\hline 1 & 570.57 & 0.0150 & 1.35 \\
2 & 535.49 & 0.0313 & 1.89 \\
3 & 391.25 & 0.6505 & 7.36 \\
5 & 377.56 & 1.0873 & 9.34 \\
7 & 350.98 & 0.7011 & 7.23 \\
8 & 344.54 & 0.3391 & 4.98 \\
13 & 312.06 & 0.0176 & 1.08 \\
17 & 305.86 & 0.0382 & 1.57 \\
18 & 305.23 & 0.0288 & 1.37 \\
23 & 296.27 & 0.0580 & 1.91 \\
24 & 293.55 & 0.1123 & 2.65 \\
30 & 284.99 & 0.0819 & 2.23 \\
31 & 275.94 & 0.0412 & 1.55 \\
38 & 249.74 & 0.1105 & 2.42 \\
52 & 228.41 & 0.0721 & 1.87 \\
61 & 218.83 & 0.0353 & 1.28 \\
65 & 217.64 & 0.0407 & 1.37 \\
\hline
\end{tabular}

Supplementary Table 2. Contributions to the $535.49 \mathrm{~nm}$ excitation.

\begin{tabular}{llc}
\hline Excitation & & X coeff. \\
\hline HOMO-1 & $\Rightarrow$ LUMO & -0.41188 \\
HOMO-1 & $\Rightarrow$ LUMO+1 & 0.47153 \\
HOMO & $\Rightarrow$ LUMO & -0.62718 \\
HOMO & $\Rightarrow$ LUMO+1 & -0.45900 \\
\hline
\end{tabular}


Supplementary Table 3. Contributions to the $377.56 \mathrm{~nm}$ excitation (Soret Band).

\begin{tabular}{llcc}
\hline Excitation & & X coeff. & Y coeff. \\
\hline HOMO-7 & $=>$ LUMO & -0.13325 & \\
HOMO-3 & $=>$ LUMO & 0.26139 & \\
HOMO-3 & $\Rightarrow$ LUMO+1 & 0.13684 & \\
HOMO-1 & $=>$ LUMO & -0.48850 & \\
HOMO-1 & $\Rightarrow$ LUMO+1 & 0.53562 & -0.11007 \\
HOMO & $\Rightarrow$ LUMO & 0.42020 & -0.10625 \\
HOMO & $=>$ LUMO+1 & 0.44151 & \\
\hline
\end{tabular}

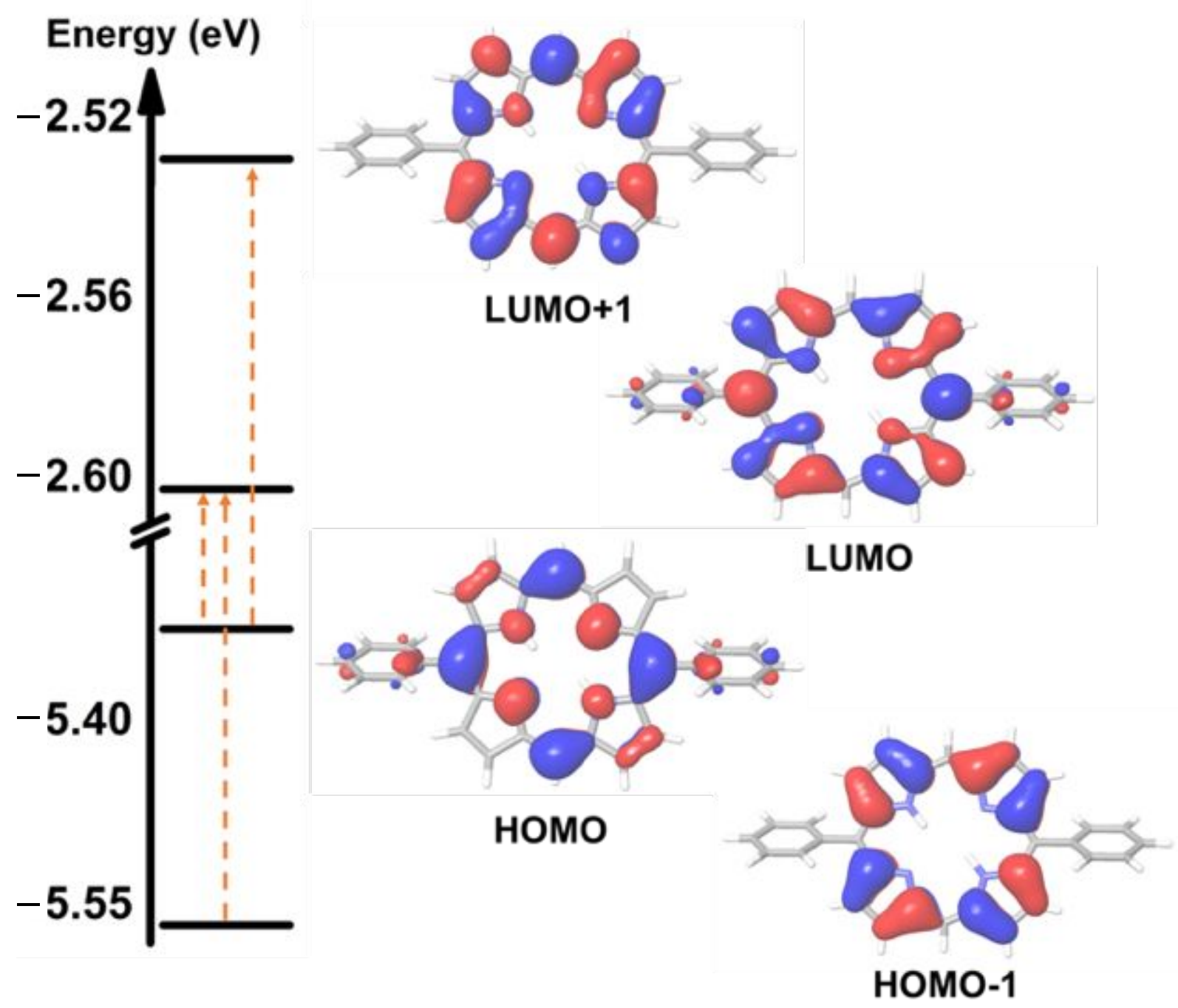

Supplementary Figure 16. Frontier orbitals of DPP 
Supplementary Table 4. RB3LYP/6-31G*+ computed transitions for $\mathbf{E x B o x}^{4+}$ DDPP with oscillator strengths $(f)$ greater than 0.015 .

\begin{tabular}{|c|c|c|c|}
\hline Transition & Wavelength(nm) & $f$ & Transition dipole moment (Debye) \\
\hline 9 & 590.15 & 0.0167 & 1.45 \\
\hline 13 & 559.11 & 0.0524 & 2.49 \\
\hline 17 & 534.31 & 0.0284 & 1.79 \\
\hline 32 & 425.65 & 0.0398 & 1.90 \\
\hline 33 & 425.17 & 0.0615 & 2.36 \\
\hline 35 & 418.26 & 0.6206 & 7.43 \\
\hline 39 & 408.28 & 0.0494 & 2.07 \\
\hline 40 & 407.84 & 0.0188 & 1.27 \\
\hline 44 & 402.35 & 0.0414 & 1.88 \\
\hline 53 & 376.90 & 0.0370 & 1.72 \\
\hline 56 & 369.41 & 0.0440 & 1.86 \\
\hline 66 & 350.95 & 0.0197 & 1.21 \\
\hline 67 & 350.82 & 0.0390 & 1.70 \\
\hline 68 & 350.09 & 0.3995 & 5.45 \\
\hline 71 & 345.67 & 0.0156 & 1.07 \\
\hline 74 & 344.42 & 0.0312 & 1.51 \\
\hline 86 & 336.27 & 0.0392 & 1.67 \\
\hline 89 & 331.71 & 0.3145 & 4.72 \\
\hline 91 & 333.04 & 0.1716 & 3.48 \\
\hline 95 & 330.80 & 0.4415 & 5.57 \\
\hline 97 & 330.44 & 0.4296 & 5.49 \\
\hline 99 & 327.90 & 0.0309 & 1.47 \\
\hline 100 & 327.15 & 0.1662 & 3.40 \\
\hline 104 & 322.20 & 0.1574 & 3.28 \\
\hline 114 & 312.58 & 0.0463 & 1.75 \\
\hline 105 & 304.13 & 0.0191 & 1.11 \\
\hline
\end{tabular}

Supplementary Table 5. Contributions to the $418.26 \mathrm{~nm}$ excitation (Soret Band). 


\begin{tabular}{llc}
\hline Excitation & & X coeff. \\
\hline HOMO-7 & $\Rightarrow$ LUMO+3 & 0.14372 \\
HOMO-5 & $\Rightarrow$ LUMO+3 & -0.42486 \\
HOMO-4 & $=$ LUMO+1 & 0.20366 \\
HOMO-4 & $\Rightarrow$ LUMO+2 & 0.20446 \\
HOMO-1 & $=$ LUMO+2 & -0.64367 \\
HOMO-1 & $\Rightarrow$ LUMO+5 & -0.30532 \\
HOMO & $\Rightarrow$ LUMO+3 & -0.40972 \\
\hline
\end{tabular}

Supplementary Table 6. Contributions to the $559.11 \mathrm{~nm}$ excitation.

\begin{tabular}{llc}
\hline Excitation & & X coeff. \\
\hline HOMO-4 & $\Rightarrow$ LUMO & -0.15199 \\
HOMO-3 & $\Rightarrow$ LUMO & 0.25033 \\
HOMO-1 & $\Rightarrow$ LUMO+1 & 0.42618 \\
HOMO-1 & $\Rightarrow$ LUMO+2 & -0.36026 \\
HOMO-1 & $\Rightarrow$ LUMO+3 & -0.18953 \\
HOMO & $\Rightarrow$ LUMO+2 & -0.35315 \\
HOMO & $\Rightarrow$ LUMO+3 & 0.65640 \\
\hline
\end{tabular}




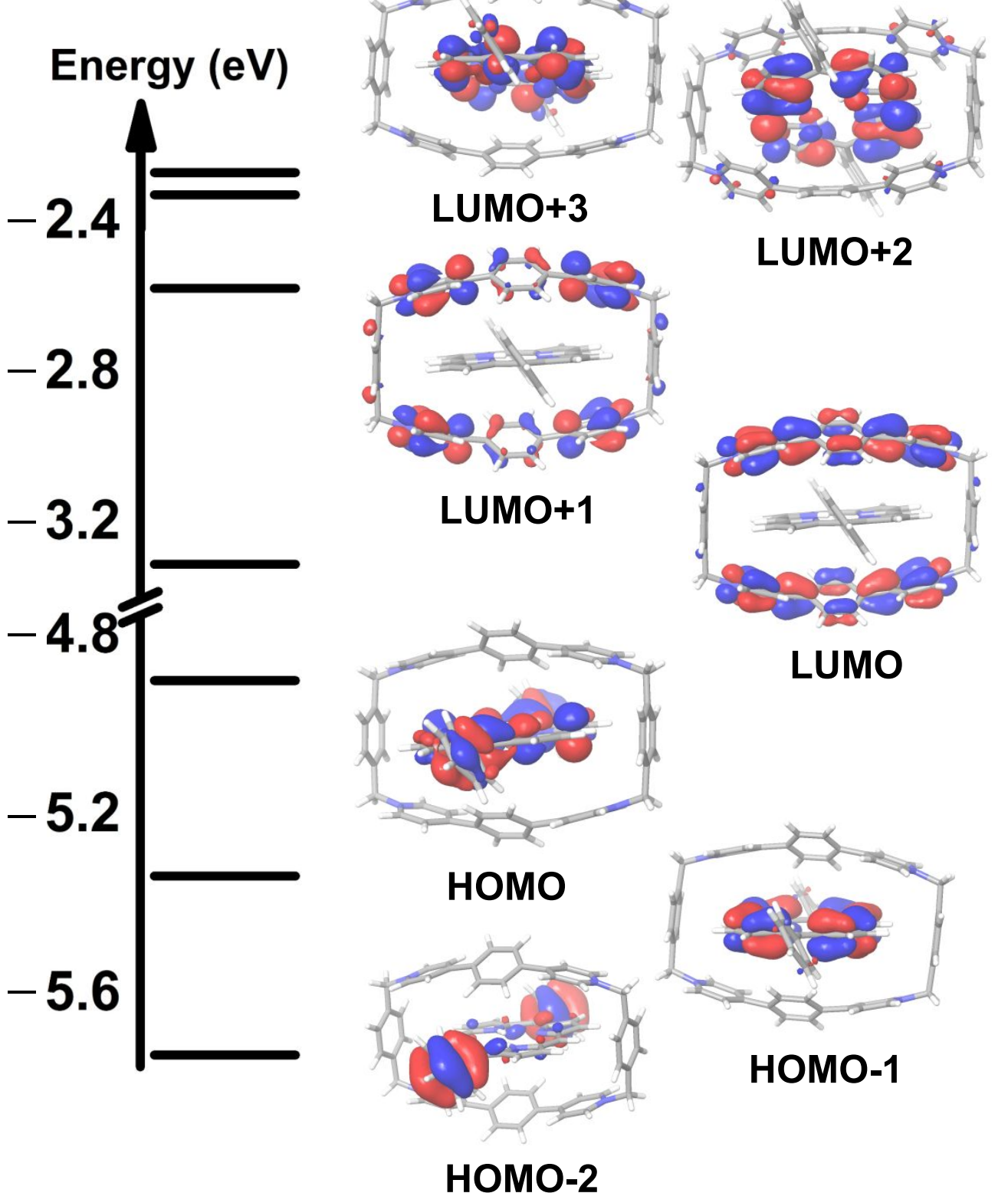

Supplementary Figure 17. Frontier orbitals of $\operatorname{ExBox}^{4+} \supset \mathrm{DPP}$

Supplementary Table 7. Frontier orbital energy levels of $\operatorname{ExBox}^{4+} \supset \mathbf{D P P}$ in $(\mathrm{eV})$

\begin{tabular}{cccccccc} 
& HOMO & HOMO-1 & HOMO-2 & LUMO & LUMO+1 & LUMO+2 & LUMO+3 \\
\hline DPP & -5.32 & -5.56 & -6.53 & -2.60 & -2.52 & -1.02 & -0.58 \\
ExBox $^{4+} \supset$ DPP & -4.89 & -5.33 & -5.73 & -3.29 & -2.59 & -2.32 & -2.26 \\
\hline
\end{tabular}




\section{Section H. Cell Experiments}

\section{Cell culture}

A2780 Cells (human ovarian cancer cell line) and MCF-7 cells (human breast adenocarcinoma cell line) obtained from Sigma-Aldrich and American Type Culture Collection (ATCC, Rockville, MD, USA), respectively, were utilized for cell culture experiments. These cells were cultured in Dulbecco's Modified Eagle's Medium (DMEM) supplemented with 10\% fetal bovine serum (FBS), penicillin $(100 \mathrm{IU} / \mathrm{mL})$ and streptomycin $(100 \mu \mathrm{g} / \mathrm{mL})$ at $37{ }^{\circ} \mathrm{C}$ in the presence of air $(95 \%)$ and $\mathrm{CO}_{2}(5 \%)$.

\section{MTT Assay}

A2780 or MCF-7 cells $\left(2 \times 10^{5}\right.$ cells/mL, $\left.100 \mu \mathrm{L}\right)$ were seeded in each well of a black 96 -well plate. A stock solution of $\mathbf{E x B o x}{ }^{4+} \supset \mathbf{D P P}$ in PBS $(1 \mathrm{mM}, 10 \mu \mathrm{L})$ was added to each well to achieve working concentrations and incubated for $12 \mathrm{~h}$. After incubation, wells were washed with PBS, to which DMEM $(100 \mu \mathrm{L})$ had been added and immediately irradiated with visible light using Max303 Xenon Light Source (385-740 nm, Asahi Spectra) at different light intensities (5-50 mW) for 1 to $15 \mathrm{~min}$. Following irradiation, cells were incubated overnight and then MTT $(5 \mathrm{mg} / \mathrm{mL}$ in PBS, $10 \mu \mathrm{L}$ ) was added into the cell media. After 4h incubation with MTT, media from each well was pipetted out, formazan crystals deposited on the plate were dissolved in of $\mathrm{Me}_{2} \mathrm{SO}(200 \mu \mathrm{L})$ and the absorbance of each well was measured using a microplate reader at $560 \mathrm{~nm}$. All the samples were analyzed in quadruplicate.

The percentage cell viability was calculated using the following formula

$\%$ cell viability $=(\mathrm{OD}$ of treated sample/ OD of untreated sample $) * 100$. 


\section{Live cell confocal microscopy}

A2780 Cells $\left(1 \times 10^{5}\right.$ cells $\left./ \mathrm{mL}, 300 \mu \mathrm{L}\right)$ were plated in each well of an 8 -well $\mu$-slide (ibidi cell focus) and cultured overnight. A PBS solution of $\mathbf{E x B o x}{ }^{4+} \supset \mathbf{D P P}$ was added to each well and incubated for predetermined time periods. For cellular co-localization studies, cells were washed with phenol-free DMEM and stained with LysoTracker green (lysosome stain, 1:1000 dilution) or MitoSpy $^{\mathrm{TM}}$ Green FM (mitochondria stain, 1:2000 dilution) or NucBlue ${ }^{\mathrm{TM}}$ Live ReadyProbes $^{\mathrm{TM}}$ Reagent (nuclei stain, 1 drop). For the live-dead assay, cells were incubated with PBS solution of $\mathbf{E x B o x}^{4+} \supset \mathbf{D P P}$ irradiated for $10 \mathrm{~min}$ at 25 or $50 \mathrm{MW}$ light intensity, incubated overnight, and stained with Calcein AM $(2 \mu \mathrm{M})$ and Ethidium homodimer-1 $(4 \mu \mathrm{M})$. Plated cells were imaged within a humidified chamber using a $63 \times$ oil-immersion objective on a SP5 Leica Confocal Microscope using HyD detectors and lasers.

\section{Intracellular ROS generation}

Intracellular ROS generation assay was performed using DCFDA Cellular ROS Detection Assay Kit (Abcam), according to the manufacturer's instructions. Briefly, A2780 cells $\left(2.5 \times 10^{5}\right.$ cells $/ \mathrm{mL}, 100 \mu \mathrm{L}$ ) were seeded in each well of a black 96-well plate and incubated with PBS solution of $\mathbf{E x B o x}{ }^{4+} \supset$ DPP for 12 h. After incubation, cells were washed with PBS and incubated with $2^{\prime}, 7^{\prime}$-dichlorofluorescin diacetate (DCFDA) $(20 \mu \mathrm{M})$ for $45 \mathrm{~min}$ in the dark. Then the cells were rinsed with PBS, irradiated and fluorescence was measured $\left(\lambda_{\mathrm{ex}}=485\right.$ and $\left.\lambda_{\mathrm{em}}=535 \mathrm{~nm}\right)$ using a microplate reader. Control experiments with PBS solution of $\mathbf{E x B o x}{ }^{4+} \supset \mathbf{D P P}$ treated cells and untreated cells, both with and without irradiation were performed.

\section{Cellular uptake studies}

A2780 cells $\left(5 \times 10^{5}\right.$ cells $\left./ \mathrm{mL}, 200 \mu \mathrm{L}\right)$ were seeded in each well of a 48 -well plate and treated

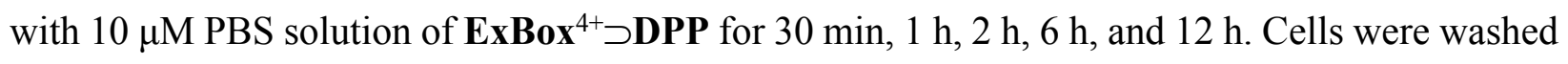


with PBS, trypsinized, and incubated with $50 \mu \mathrm{L}$ of 1:100 Zombie Aqua fixable cell viability dye for $15 \mathrm{~min}$ at $4{ }^{\circ} \mathrm{C}$. After incubation, cells were washed with PBS $(600 \mu \mathrm{L})$, spun at $400 \operatorname{rcf}$ for 5 min, and the supernatant discarded. Cells were then suspended in $2 \%$ paraformaldehyde $(100 \mu \mathrm{L})$ prior to being analyzed using a BD Fortessa flow cytometer. Cells were first gated for singlet events using FSC-A vs. FSC-H, after which debris was excluded used FSC-A vs. SSC-A. Cells gated as Zombie Aqua low were considered live cells, which were then analyzed for their median fluorescence intensity (MFI) in the PE-Cy5 channel for $\mathbf{E x B o x}{ }^{4+} \supset$ DPP fluorescence, representing the amount of $\mathbf{E x B o x}^{4+} \supset$ DPP taken up by each cell.

\section{Apoptosis assay}

A2780 Cells $\left(5 \times 10^{5}\right.$ cells $\left./ \mathrm{mL}, 100 \mu \mathrm{L}\right)$ were seeded in each well of a Black 96-well plate and incubated with 5 or $10 \mu \mathrm{M}$ PBS solution of $\mathbf{E x B o x}{ }^{4+} \supset$ DPP for $12 \mathrm{~h}$. After incubation, cells were washed with PBS, DMEM $(100 \mu \mathrm{L})$ had been added and immediately irradiated with visible light using Max-303 Xenon Light Source (385-740 nm, Asahi Spectra) at 25 and $50 \mathrm{~mW}$ light intensities for $10 \mathrm{~min}$. After irradiation, cells were incubated for an additional $3 \mathrm{~h}$ prior to being trypsinized and recovered for annexin V staining. Annexin V solution (1:50 FITC Annexin V in Annexin V staining buffer, $50 \mu \mathrm{L}$ ) was added to cells and was incubated at room temperature for $20 \mathrm{~min} .1 \times$ PBS $(500 \mu \mathrm{L})$ was then added to cells, which were centrifuged at $400 \mathrm{rcf}$ for $5 \mathrm{~min}$, and the supernatant was discarded. Cells were resuspended in 1:20 7-AAD $(100 \mu \mathrm{L})$ in cell staining buffer and were incubated for 10 min prior to analysis on a BD Fortessa flow cytometer. Cells were first gated for singlet events using FSC-A vs. FSC-H, after which debris was excluded used FSC-A vs. SSC-A. All remaining events were gated as live, dead, or apoptotic based on annexin V staining vs. 7-AAD staining, with dead cells staining 7-AAD+, live cells staining as 7-AAD- and annexin$\mathrm{V}-$, and apoptotic cells staining 7-AAD-annexin- $\mathrm{V}+$. Cells without irradiation were used as controls. 


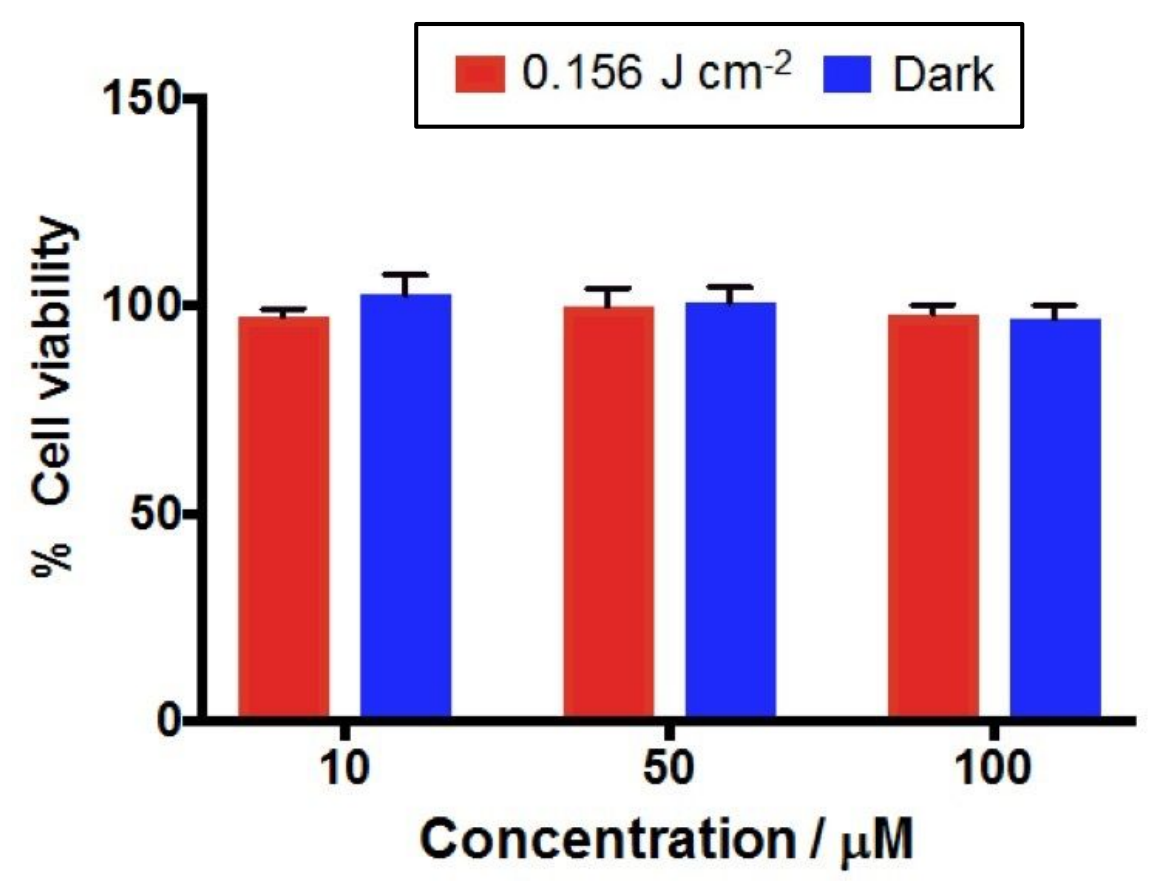

Supplementary Figure 18. A2780 Cell viability at different concentrations of $\mathbf{E x B o x}^{4+}$ with or without (dark) irradiation (light dose $=0.156 \mathrm{~J} \mathrm{~cm}^{-2}$ ) for $10 \mathrm{~min}$. Cell viability was measured using MTT assay. Error bars represent SD, $n=4$.

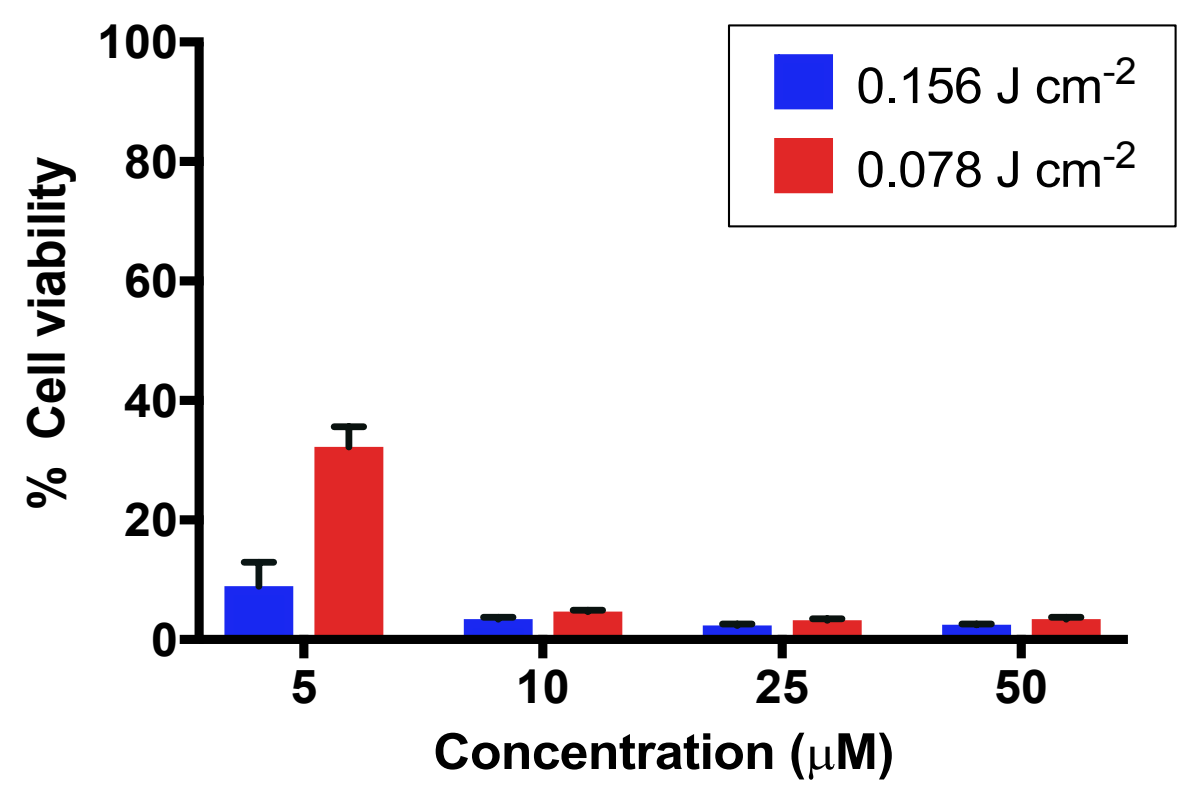

Supplementary Figure 19. A2780 cell viability after treatment with different concentrations of $\mathbf{D P P H}_{2}{ }^{+2}$ in PBS and irradiation with different light doses for $10 \mathrm{~min}$. Cell viability was measured using the MTT assay. Error bars represent SD, $\mathrm{n}=4$. 


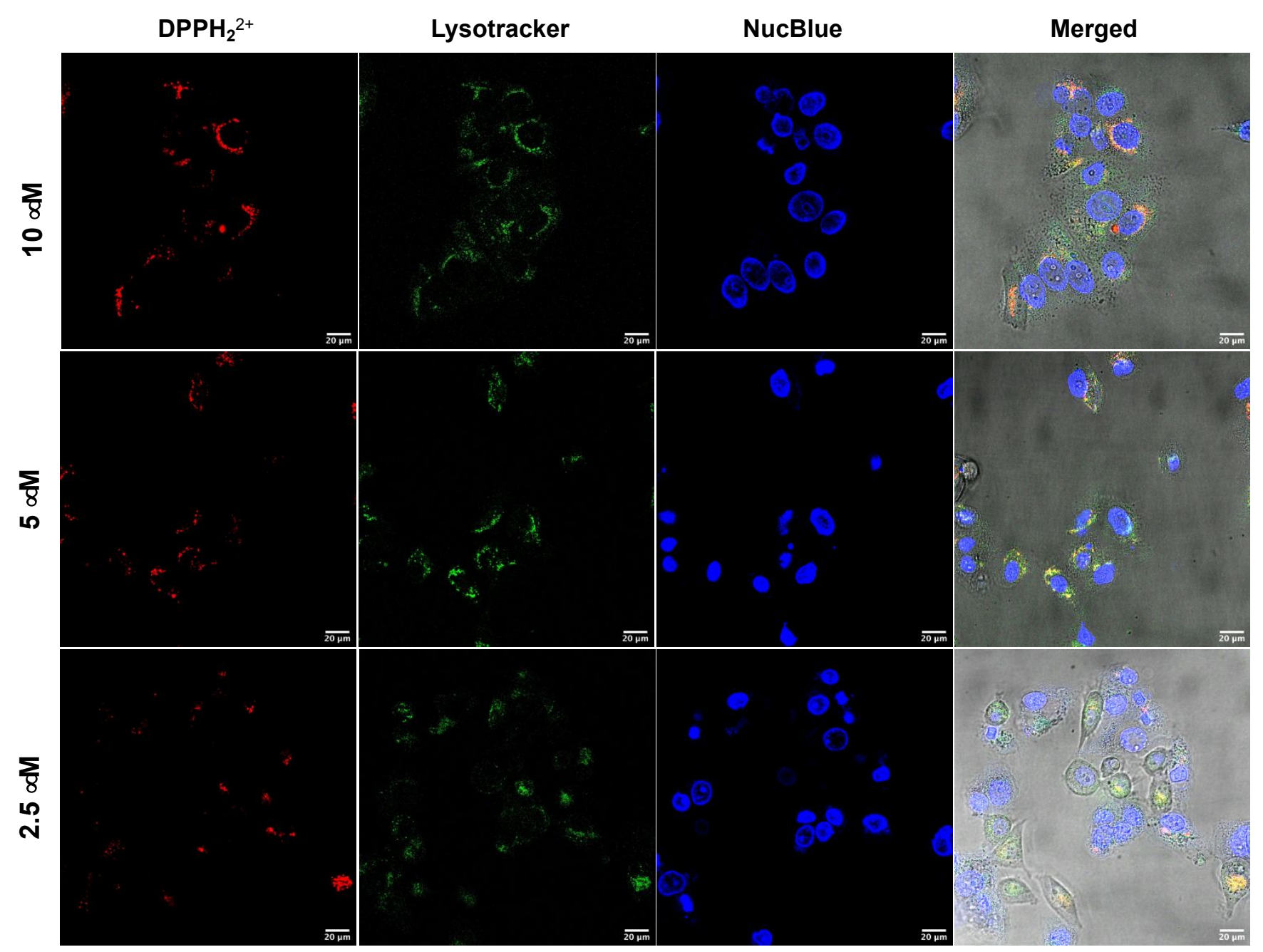

Supplementary Figure 20. Concentration-dependent uptake of $\mathbf{D P P H}_{2}{ }^{2+}$. Live-cell confocal microscopy images of A2780 cells stained with Lysotracker green, NucBlue and $\mathbf{D P P H}{ }_{2}{ }^{2+}$ showing lysosomal co-localization of $\mathbf{D P P H} \mathbf{H}^{+2}$ after incubation for $12 \mathrm{~h}$ with A2780 cells. Scale bar is $20 \mu \mathrm{m}$. 
(a)

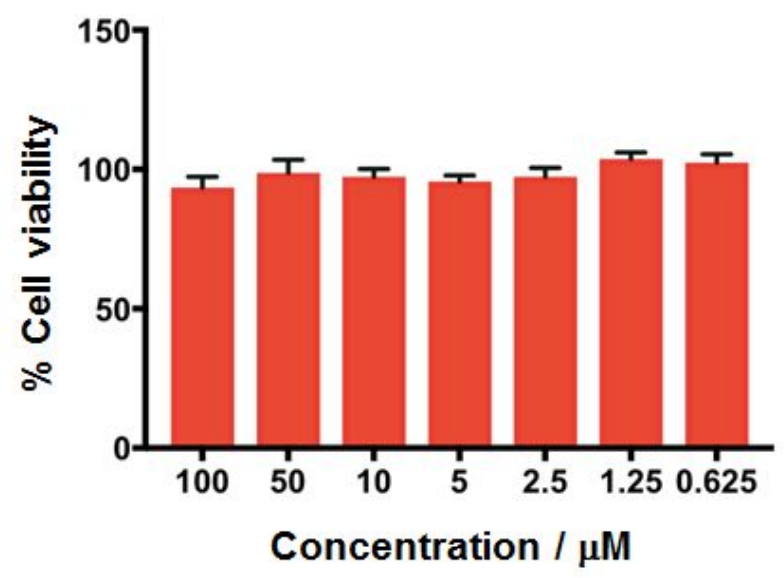

(b)

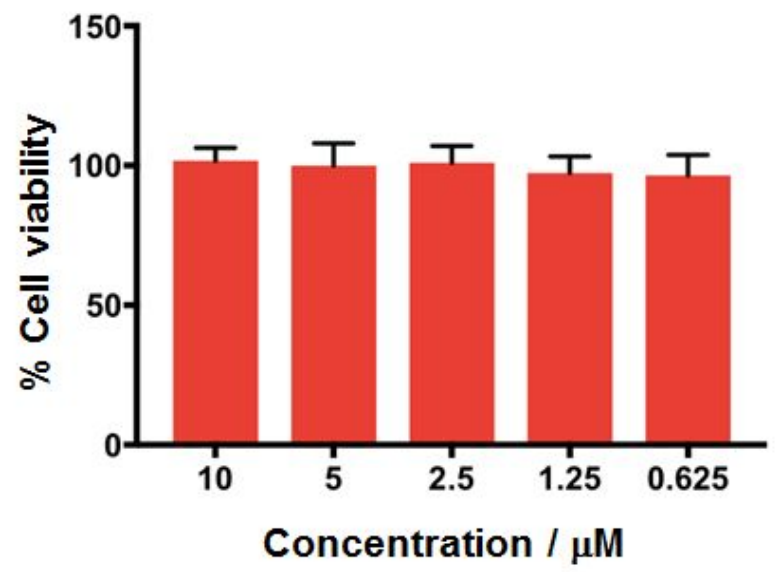

Supplementary Figure 21. (a) A2780 and (b) MCF-7 cell viability after treatment with different concentrations of $\mathbf{E x B o x}^{4+} \supset$ DPP in PBS in the dark. Cell viability was measured using the MTT assay. Error bars represent SD, $n=4$.
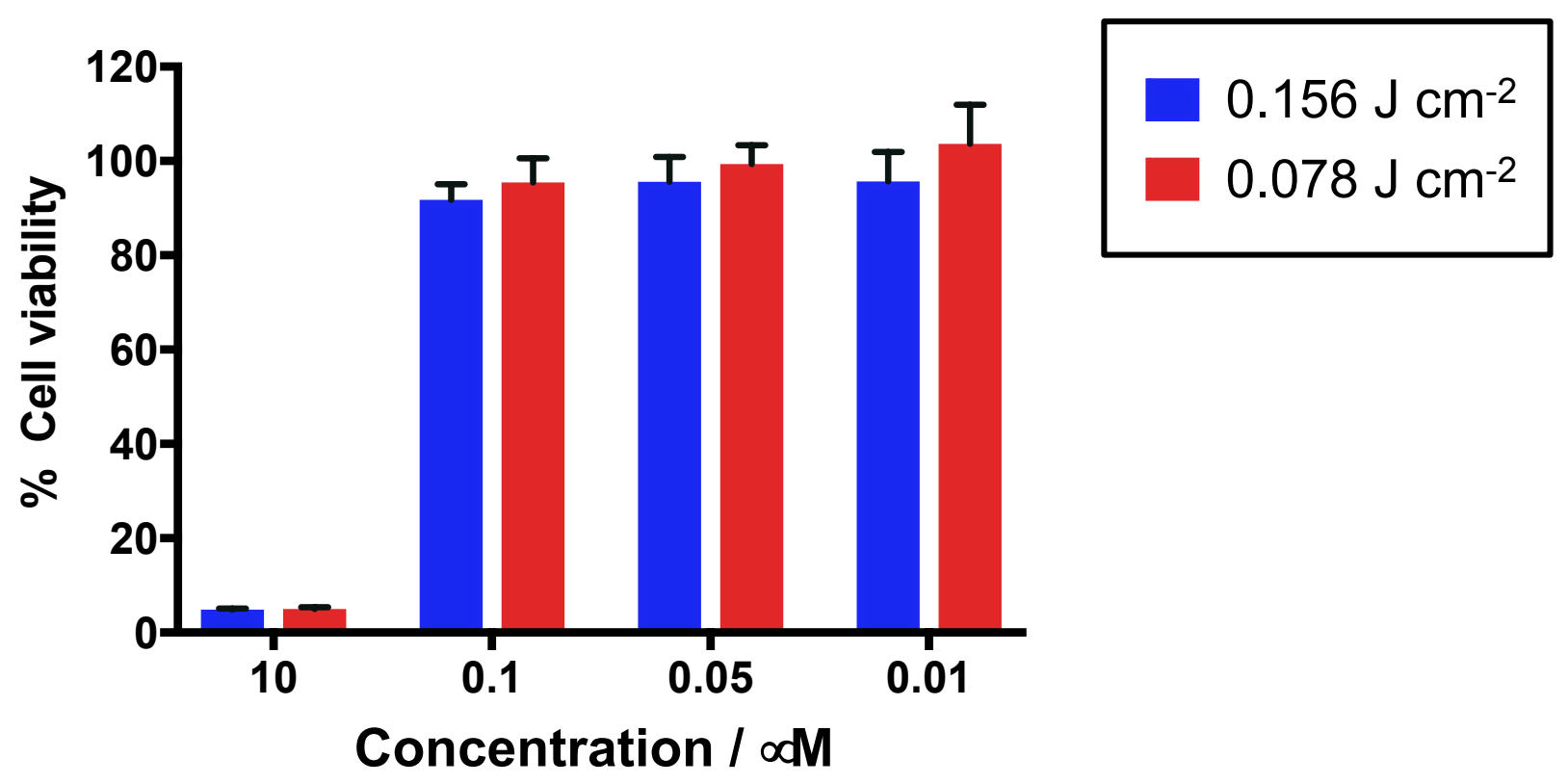

Supplementary Figure 22. A2780 cell viability after treatment with different concentrations of $\mathbf{E x B o x}^{4+} \supset \mathbf{D P P}$ in PBS and light doses of 0.078 and $0.156 \mathrm{~J} \mathrm{~cm}^{-2}$ for $10 \mathrm{~min}$. Cell viability was measured using MTT assay. Error bars represent SD, $n=4$. 

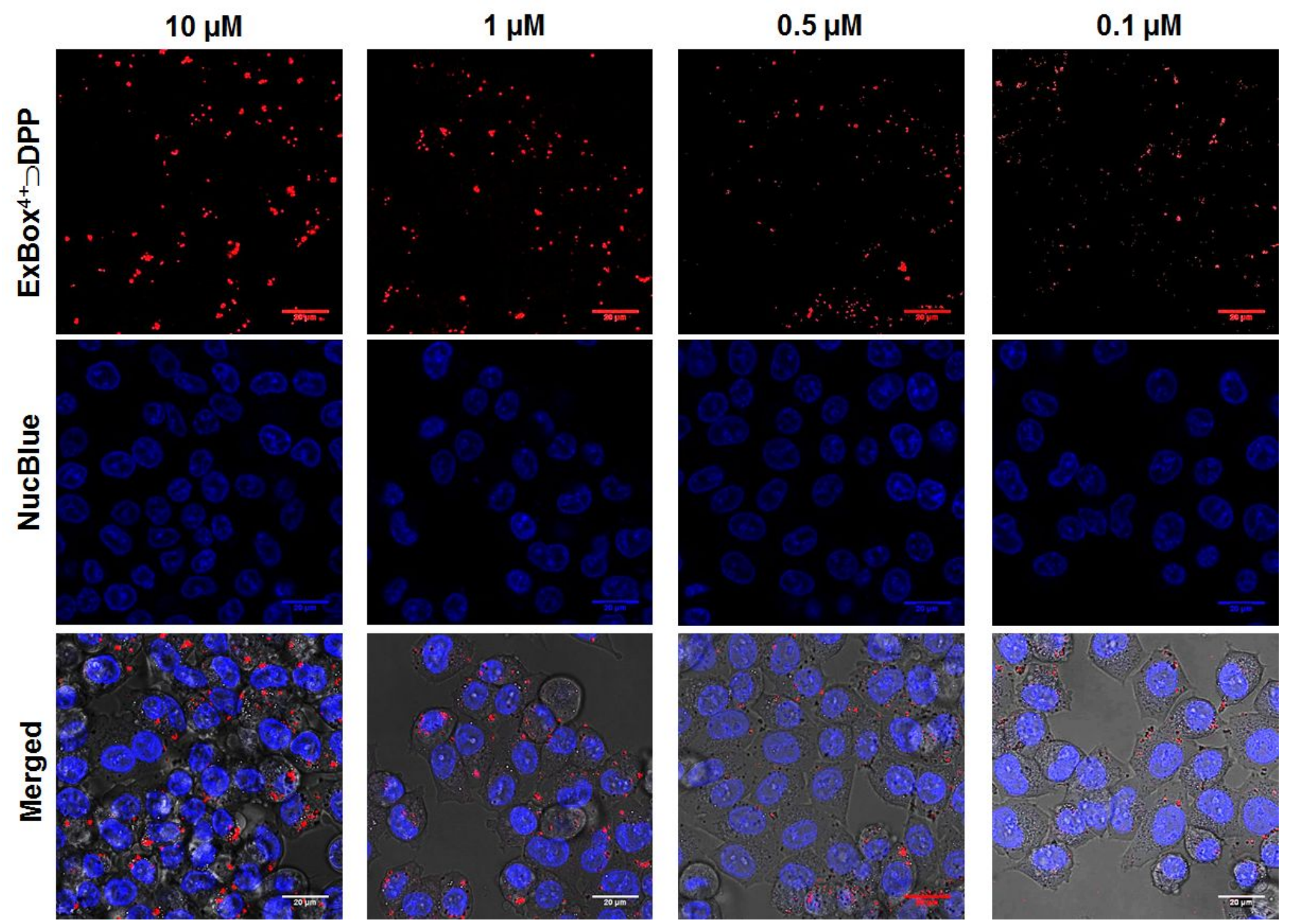

Supplementary Figure 23. Concentration dependent uptake of ExBox ${ }^{4+} \supset$ DPP. Live-cell confocal microscopy images of A2780 cells stained with NucBlue ${ }^{\circledR}$ Live Cell Stain (blue) following incubation with different concentrations $(10,1,0.5$, and $0.1 \mu \mathrm{M})$ of $\mathbf{E x B o x}^{4+} \supset \mathbf{D P P}(\mathrm{red})$ in PBS for $12 \mathrm{~h}$. Scale bar is $20 \mu \mathrm{m}$. 

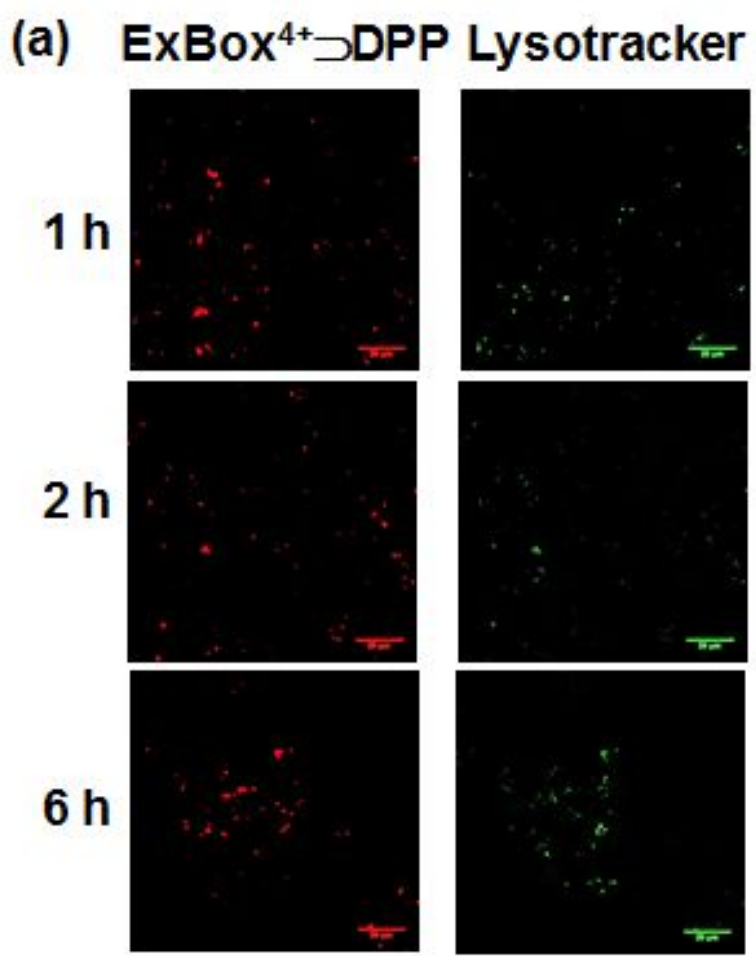

$12 \mathrm{~h}$

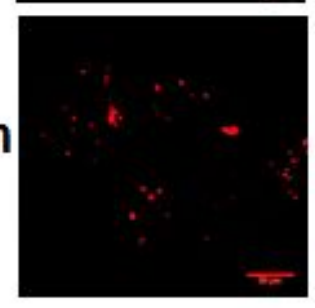

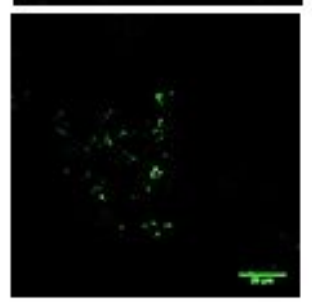
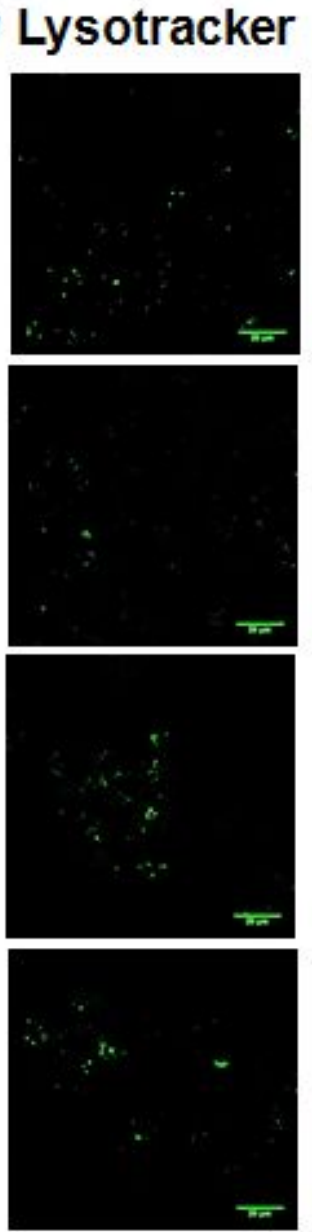

NucBlue
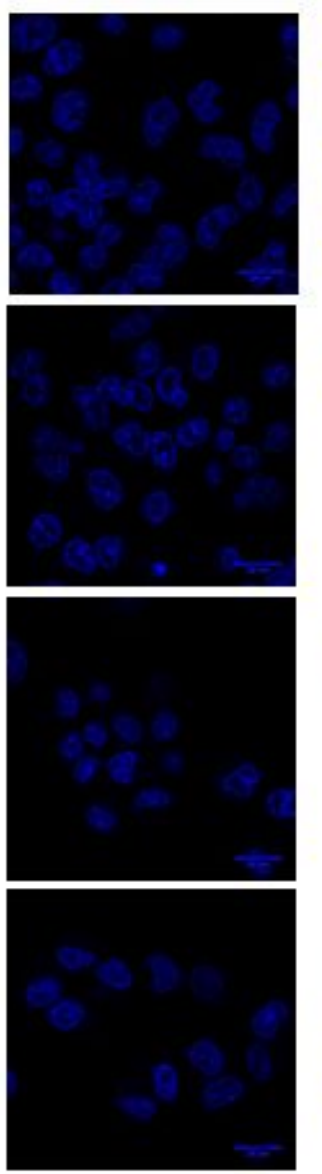

Merged
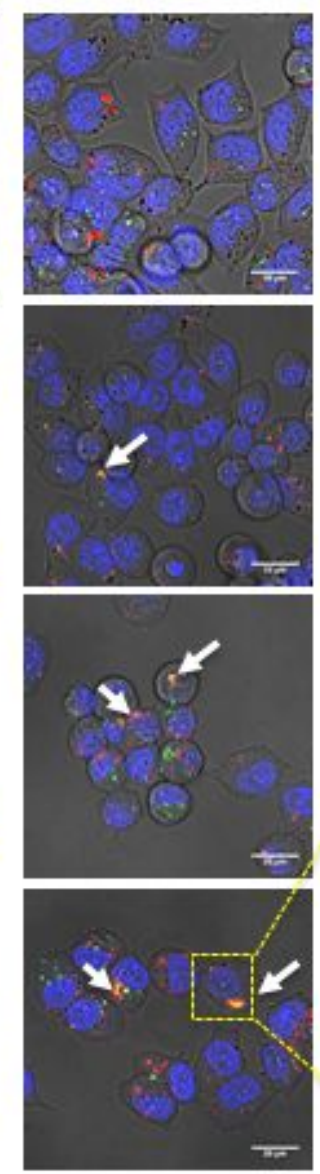

(c)

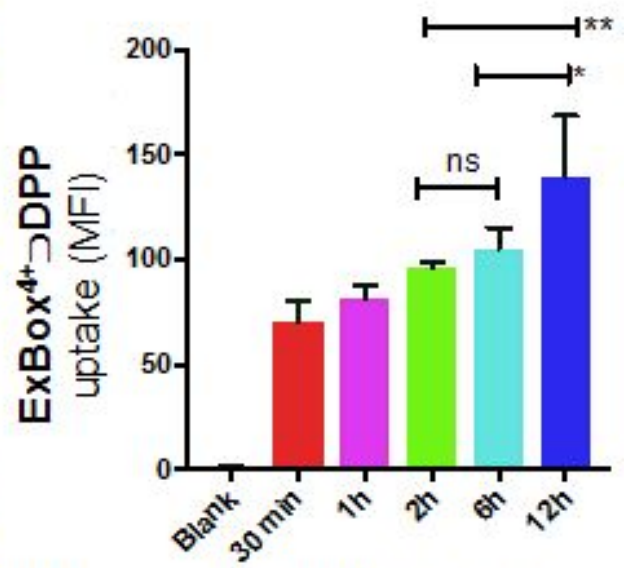

(b) Incubation Time

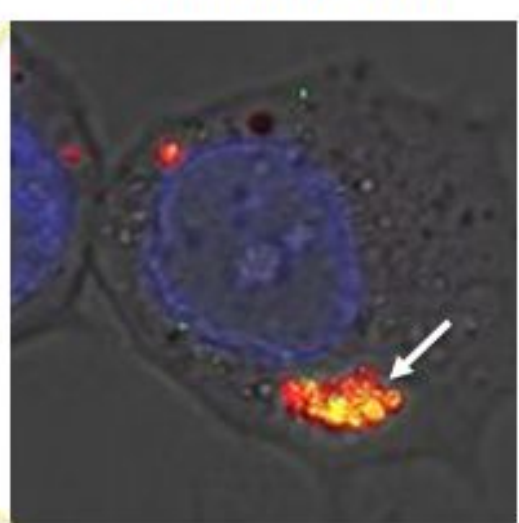

Supplementary Figure 24. (a) Live-cell confocal microscopy images of A2780 cells stained with Lysotracker green and NucBlue showing endolysosomal co-localization of $\mathbf{E x B o x}{ }^{4+} \supset \mathbf{D P P}$ after incubation for 1, 2, 6, and $12 \mathrm{~h}$ with A2780 cells. White arrows in the merged images

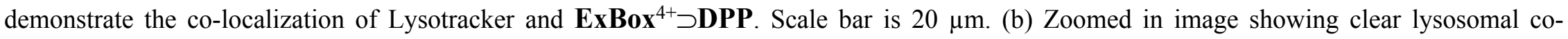

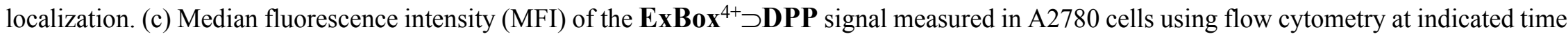
points. Results denoted are mean MFI value $\pm \mathrm{SD}, \mathrm{n}=4$. Significance determined by one-way ANOVA and Tukey's multiple comparisons test. 

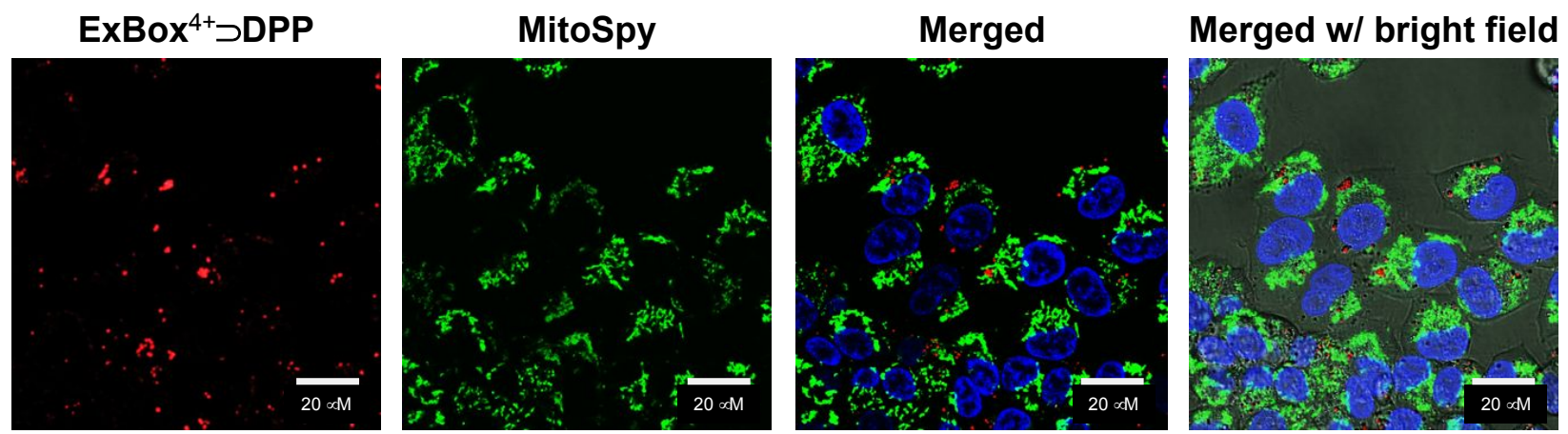

Supplementary Figure 25. Live-cell confocal microscopy images of A2780 cells stained with nuclei stain NucBlue ${ }^{\circledR}$ (blue) and mitochondrial staining dye MitoSpy ${ }^{\mathrm{TM}}$ Green FM (green) following incubation with $10 \mu \mathrm{M}$ of $\mathbf{E x B o x}{ }^{4+} \supset \mathbf{D P P}$ (red) in PBS for $12 \mathrm{~h}$. Scale bar is $20 \mu \mathrm{m}$.

(a)

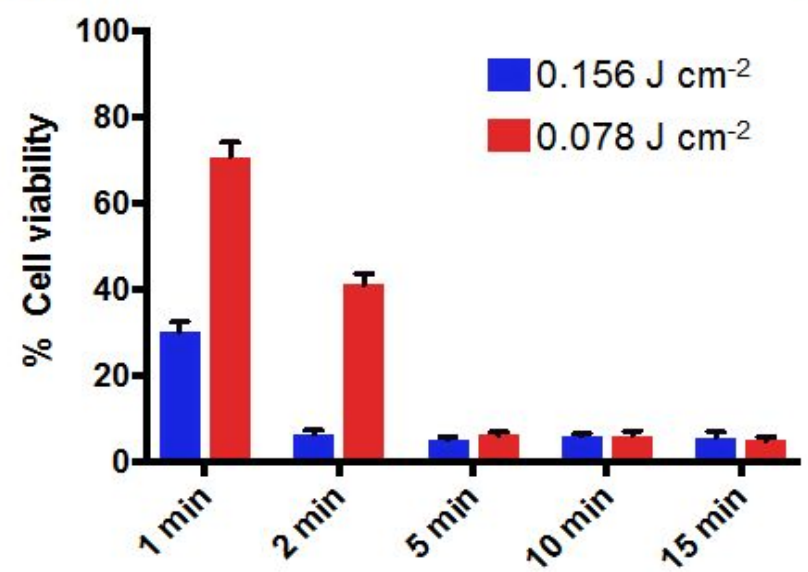

(b)

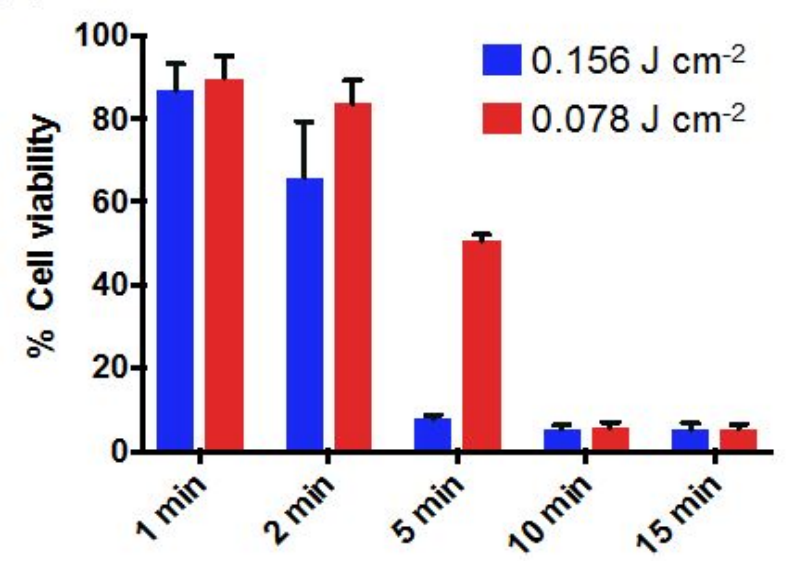

Supplementary Figure 26. Effect of irradiation time on A2780 cell viability after treatment with (a) $10 \mu \mathrm{M}$ and (b) $5 \mu \mathrm{M} \mathbf{E x B o x}{ }^{4+} \supset \mathbf{D P P}$ in PBS. Error bars represent SD, $\mathrm{n}=4$. 


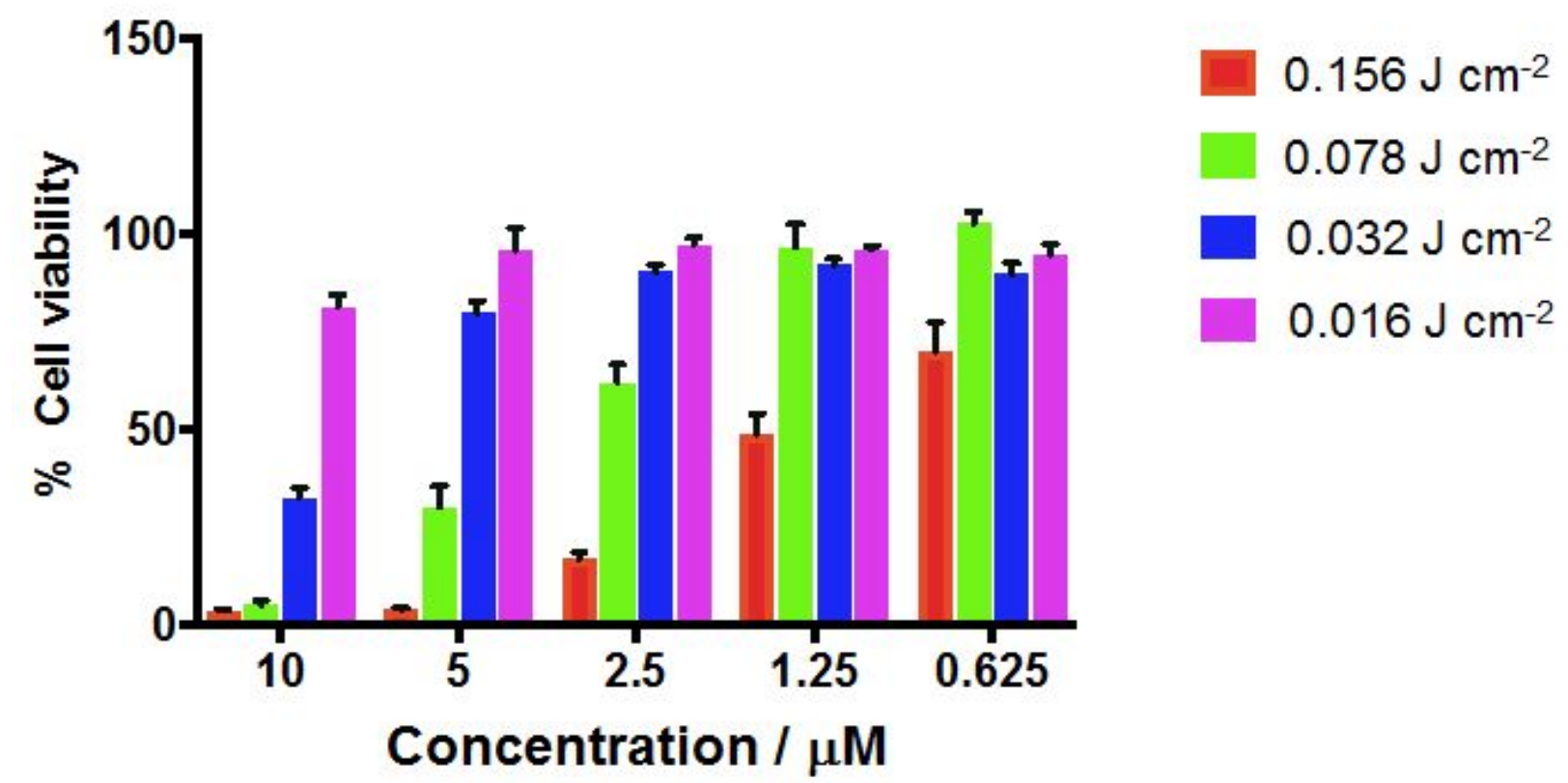

Supplementary Figure 27. MCF-7 cell viability after treatment with different concentrations of $\mathbf{E x B o x}^{4+} \supset$ DPP in PBS and irradiation with different light doses for 10 min. Cell viability was measured using MTT assay. Error bars represent SD, $\mathrm{n}=4$.

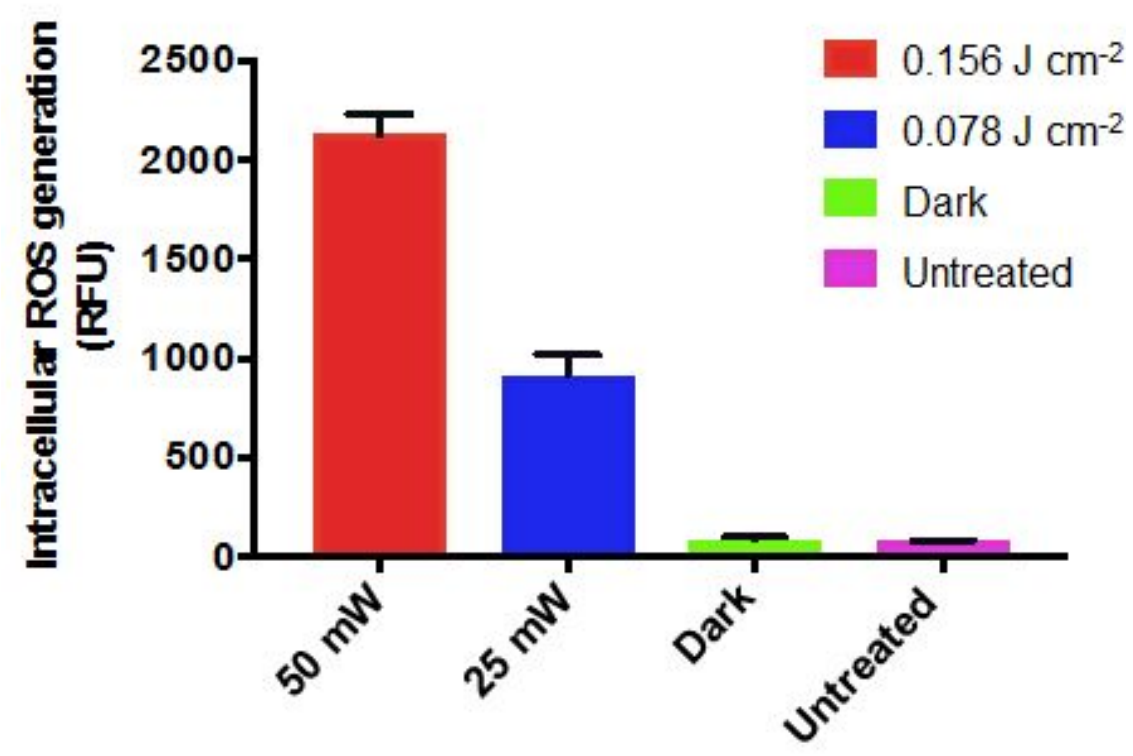

Supplementary Figure 28. Quantitative intracellular ROS generation in A2780 cells treated with $\operatorname{ExBox}^{4+} \supset \mathbf{D P P}(10 \mu \mathrm{M})$, exposed to light dose of 0.078 and $0.156 \mathrm{~J} \mathrm{~cm}^{-2}$ or kept in the dark. Error bars represent $\mathrm{SD}, \mathrm{n}=4$. 
(a)

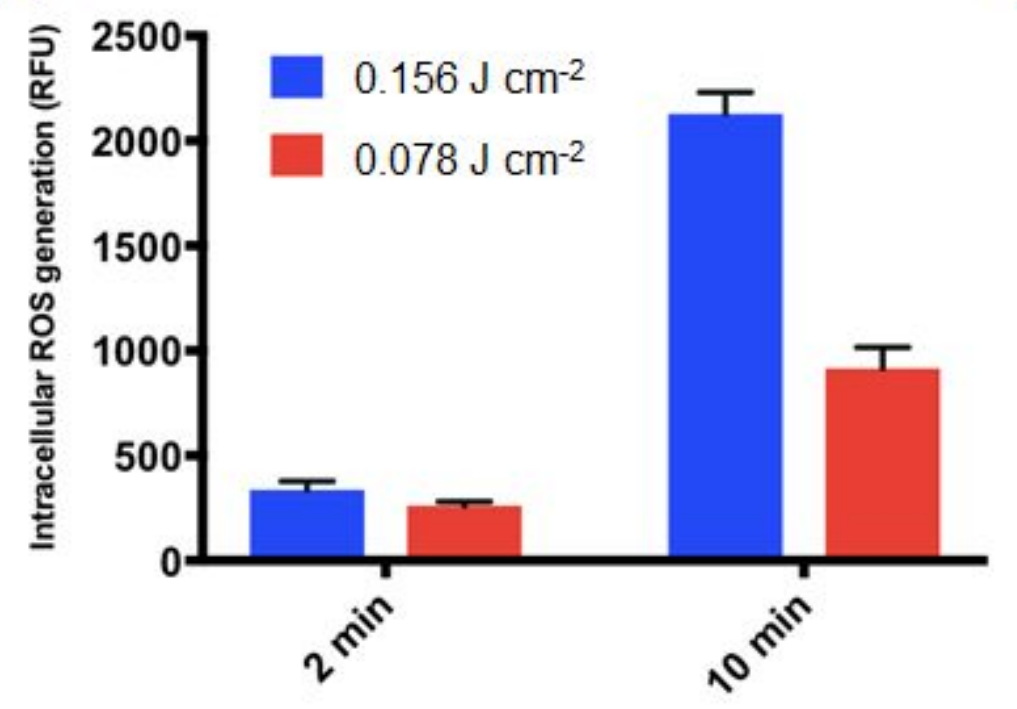

(b)

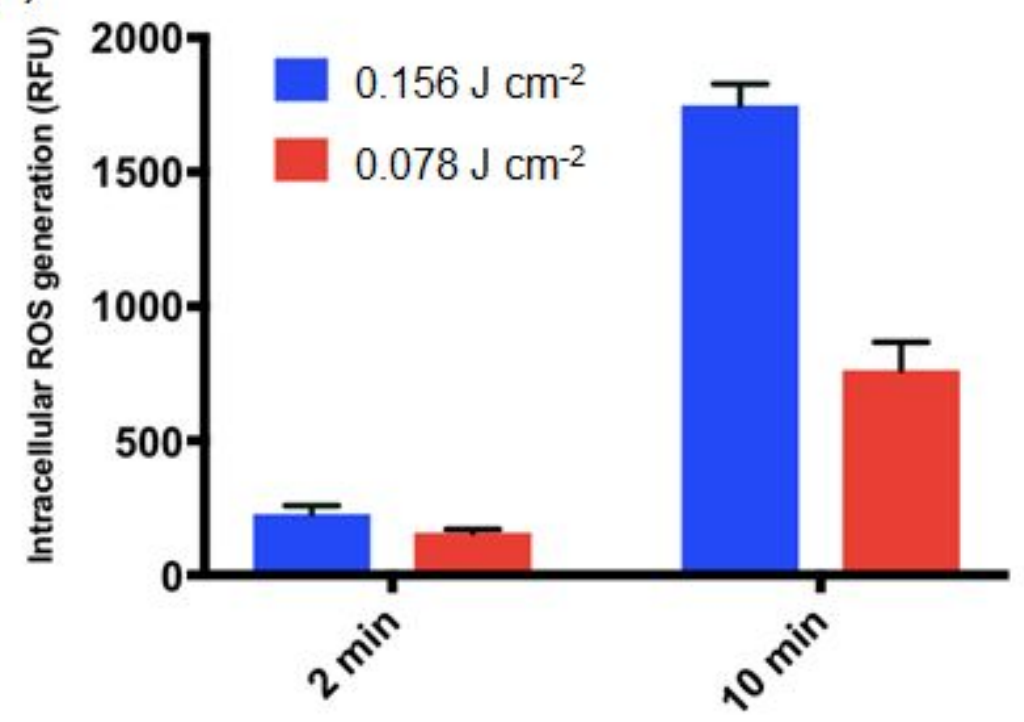

Supplementary Figure 29. Effect of irradiation time on ROS generation in A2780 cells after treatment with (a) $10 \mu \mathrm{M}$ and (b) $5 \mu \mathrm{M}$ $\mathbf{E x B o x}^{4+} \supset$ DPP. Error bars represent SD, $n=4$. 


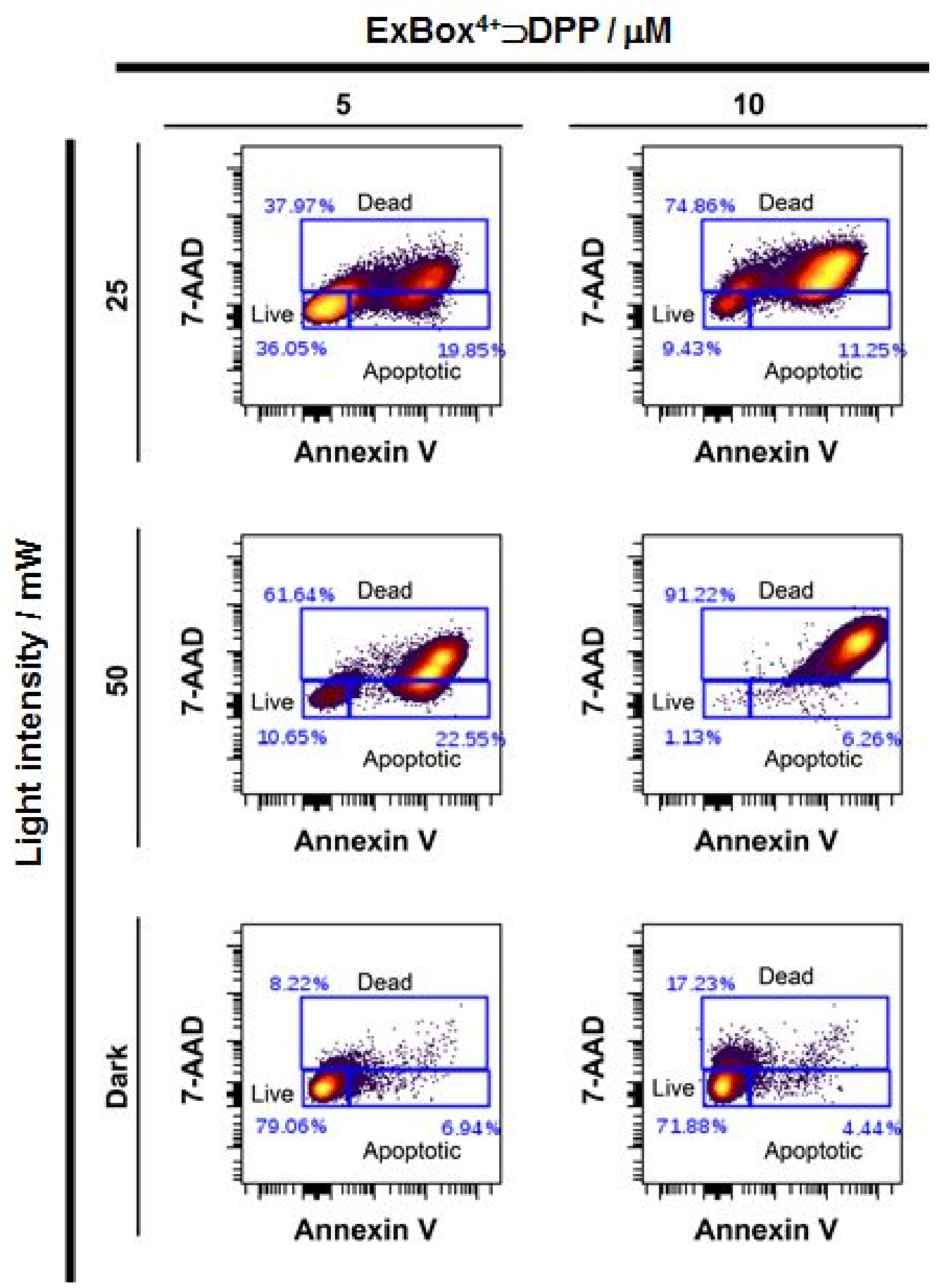

Supplementary Figure 30. Representative Annexin V Apoptosis flow assay contour plots of

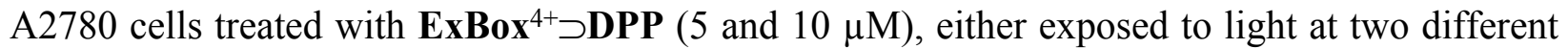
intensities (25 and $50 \mathrm{~mW}$ ) or kept in the dark. Gates are shown as blue boxes with values (in blue) representing the percentage of events within each gate. X-Axis represents the Annexin V FITC fluorescence intensity, and the $\mathrm{Y}$-axis represents the 7-AAD fluorescence intensity. 


\section{Section I. References}

(1) The MathWorks, Inc., Natick, Massachusetts, United States.

(2) Berberan-Santos, M. N.; Martinho, J. M. G. The integration of kinetic rate equations by matrix methods J. Chem. Educ., 1990, 67, 375-379.

(3) Barnes, J. C.; Juríček, M.; Strutt, N. L.; Frasconi, M.; Sampath, S.; Giesener, M. A.; McGrier, P. L.; Bruns, C. J.; Stern, C. L.; Sarjeant, A. A.; Stoddart, J. F. ExBox: A polycyclic aromatic hydrocarbon scavenger. J. Am. Chem. Soc. 2013, 135, 183-192.

(4) Dolomanov, O. V.; Bourhis, L. J.; Gildea, R. J.; Howard, J. A. K.; Puschmann, H. OLEX2: A complete structure solution, refinement and analysis program. J. Appl. Cryst. 2009, 42, 339341.

(5) Sheldrick, G. M. SHELXT-Integrated space-group and crystal-structure determination. Acta Cryst. Sect. A 2015, 71, 3-8.

(6) Sheldrick, G. M. A short history of SHELX. Acta Cryst. A 2008, 64, 112-122.

(7) Bochevarov, A. D.; Harder, E.; Hughes, T. F.; Greenwood, J. R.; Braden, D. A.; Philipp, D. M.; Rinaldo, D.; Halls, M. D.; Zhang, J.; Friesner, R. A. Jaguar: A high-performance quantum chemistry software program with strengths in life and materials scisences. Int J Quantum Chem. 2013, 113, 2110-2142. 